\title{
OMEGA LIMITS, PROLONGATIONAL LIMITS AND ALMOST PERIODIC POINTS OF A CONTINUOUS FLOW VIA EXTERIOR SPACES
}

\author{
José Manuel García Calcines, Luis Javier Hernández Paricio and \\ María Teresa Rivas Rodríguez \\ Universidad de La Laguna and Universidad de La Rioja, Spain
}

Abstract. In this paper we analyse some applications of the category of exterior spaces to the study of dynamical systems (flows). The limit space and end space of an exterior space are used to construct different types of limit spaces and end spaces of a dynamical system. In this work we analyse the relationships between the notions and constructions given by the exterior structures of a continuous flow and the more usual notions of omega-limits, first prolongational limits and several types of almost periodic points (Poisson-stable points, non-wandering points) of a flow

\section{INTRODUCTION}

Recently the use of the category of exterior spaces has been applied to give a new approach to the study of continuous and discrete dynamical systems, see $[19,20]$. This paper contains new results about the relations and applications of the category of exterior spaces to the study of continuous flows.

The category of exterior spaces has been provided with a well developed homotopy theory $([12,16-18,21])$. The study of the exterior and proper homotopy invariants has proved to be useful in the study of non-compact manifolds $([8,30])$, the study of the shape of some compact spaces ([22]), the L-S proper category $([14,15])$, et cetera.

2010 Mathematics Subject Classification. 54H20, 37B99.

Key words and phrases. Dynamical system, exterior space, exterior flow, limit space functor, end space functor, Freudenthal end point, periodic point, agglomerative point, Poisson-stable point, omega limit, region of attraction, attractor, repeller, non wandering point, first prolongational limit of a point, Lagrange-stable point, dispersive flow, basin of an end point. 
On the one hand, this category of exterior spaces has its own notions and constructions and others inherited from the category of topological spaces and from categories of pro-spaces ([10]). For example, for categories of pro-spaces one can consider the inverse limit of a prospace and homotopy invariants such as homotopy pro-groups and Steenrod, Čech and Brown-Grossmann groups (we note that the zero-dimensional Cech invariant is the set of Freudenthal ends when we consider the inverse system of cocompact subsets in a topological space).

Let us recall the definition of exterior space and some basic constructions (in this paper the exterior concepts and constructions are typed in boldface): Let $\left(X, \mathbf{t}_{X}\right)$ be a topological space, where $\mathbf{t}_{X}$ denotes the topology of $X$. An externology on $\left(X, \mathbf{t}_{X}\right)$ is a non-empty collection $\varepsilon(X)$ of open subsets which is closed under finite intersections and such that if $E \in \varepsilon(X), U \in \mathbf{t}_{X}$ and $E \subset U$, then $U \in \varepsilon(X)$. The members of $\varepsilon(X)$ are called exterior open subsets. An exterior space $X=\left(X, \varepsilon(X), \mathbf{t}_{X}\right)$ consists of a topological space $\left(X, \mathbf{t}_{X}\right)$ together with an externology $\varepsilon(X)$. The limit space of an exterior space $X$ is the topological subspace $L(X)=\cap_{E \in \varepsilon(X)} E$ and the barlimit space of $X$ is the topological subspace $\bar{L}(X)=\cap_{E \in \varepsilon(X)} \bar{E}$, where $\bar{E}$ denotes the closure of $E$.

On the other hand, we have common dynamical notions (which are typed in italics) as the following: A flow on a topological space $X=\left(X, \mathbf{t}_{X}\right)$ is a continuous map $\varphi: \mathbb{R} \times X \rightarrow X, \varphi(t, x)=t \cdot x$, such that $0 \cdot x=x, \forall x \in X$ and $t \cdot(s \cdot x)=(t+s) \cdot x, \forall x \in X, \forall t, s \in \mathbb{R}$. A point $x \in X$ is said to be periodic if there is $t \in \mathbb{R}, t \neq 0$ such that $t \cdot x=x$. We denote by $P(X)$ the invariant subset of periodic points of $X$. For a flow $X=(X, \varphi)$, the $\Lambda^{+}$-limit set (or omega-limit set) of a point $x \in X$ is given as follows: $\Lambda^{+}(x)=\left\{y \in X \mid \exists\right.$ a net $t_{\delta} \rightarrow+\infty$ such that $\left.t_{\delta} \cdot x \rightarrow y\right\}$. The $\Lambda^{+}$-limit set of a flow $X$ is the following invariant subset: $\Lambda^{+}(X)=\bigcup_{x \in X} \Lambda^{+}(x)$.

The main goal of this paper is to find the most important relationships between the exterior notions and constructions and dynamic concepts.

To find the connections between exterior and dynamic concepts we construct a hybrid one containing both notions. We proceed as follows: Let $\left(X, \mathbf{t}_{X}\right)$ be a topological space endowed with an additional topology $\tau$ satisfying that $\mathbf{t}_{X} \subset \tau \subset \mathbf{d}_{X}$, where $\mathbf{d}_{X}$ is the discrete topology. Denote by $\tau_{x}=\{U \in \tau \mid x \in U\}$, where $x \in X$. On $\mathbb{R}$ with the usual topology we consider three externologies $\{\mathbf{r}, \mathbf{l}, \mathbf{c}\}$ which are induced by the neighbourhoods at $+\infty,-\infty, \infty$ (the Alexandroff infinity), respectively. Now given a pair $(\epsilon, \tau)$ with $\epsilon \in\{\mathbf{r}, \mathbf{l}, \mathbf{c}\}$ and $\mathbf{t}_{X} \subset \tau \subset \mathbf{d}_{X}$ and a flow $\varphi: \mathbb{R} \times X \rightarrow X$, an open $N \in \mathbf{t}_{X}$ is said to be $(\epsilon, \tau)$-exterior if for any $x \in X$ there are $T^{x} \in \epsilon$ and $U_{x} \in \tau_{x}$ such that $\varphi\left(T^{x} \times U_{x}\right) \subset N$. It is easy to check that the family of $(\epsilon, \tau)$-exterior subsets is an externology, that will be denoted by $\varepsilon^{(\epsilon, \tau)}(X)$. Therefore, we 
have the exterior flow $X^{(\epsilon, \tau)}=\left(X, \varepsilon^{(\epsilon, \tau)}(X), \varphi\right)$. For concepts related to an exterior flow we will use bold italics.

In this paper we show the existence of many relationships between exterior and dynamic theories. For instance, taking $\tau=\mathbf{d}_{X}$, for a $T_{1}$ flow $X$, we have proved (Theorem 6.3) that the limit space is the subflow of periodic points:

$$
L\left(X^{\left(\epsilon, \mathbf{d}_{X}\right)}\right)=P(X),
$$

and Corollary 7.6 (see also Definition 4.1) shows that under some topological conditions the bar-limit space is the topological closure of the $\Lambda^{+}$-limit set of $X$ :

$$
\bar{L}\left(X^{\left(\mathbf{r}, \mathbf{d}_{X}\right)}\right)=\overline{\Lambda^{+}(X)} .
$$

We note that the limit and bar-limit constructions developed for exterior spaces, which appear on the left side of the equalities above, can be interpreted as relevant subflows of a dynamical system.

In our study we also analyse these types of relationships between other exterior concepts and the more usual notions of omega-limits, first prolongational limits and several types of almost periodic points (Poisson-stable points, non-wandering points) of a flow.

It is worth pointing out that when we take $\tau=\mathbf{t}_{X}$ the corresponding exterior notions are related to dynamical notions which depend on the local behavior of the dynamical system. In this paper we analyse six externologies which correspond to the six pairs $(\epsilon, \tau) \in\{\mathbf{r}, \mathbf{l}, \mathbf{c}\} \times\left\{\mathbf{t}_{X}, \mathbf{d}_{X}\right\}$. Given a point $x$ in a flow $X$ and an open neighbourhood $U$ at $x$, the forward behavior of the trajectory at $x\left(\left(t_{0},+\infty\right) \cdot x\right)$ is related to the externology $\varepsilon^{\left(\mathbf{r}, \mathbf{d}_{X}\right)}(X)$ and, in order to study the forward behavior of an open neighbourhood $U$ at $x\left(\left(t_{0},+\infty\right) \cdot U, U \in \mathbf{t}_{X_{x}}\right)$, we consider the externology $\varepsilon^{\left(\mathbf{r}, \mathbf{t}_{X}\right)}(X)$. The backward behavior of a point $x$ or an open neighbourhood $U$ is connected with the properties of the externologies $\varepsilon^{\left(\mathbf{l}, \mathbf{d}_{X}\right)}(X), \varepsilon^{\left(\mathbf{l}, \mathbf{t}_{X}\right)}(X)$, respectively. In order to simultaneously analyse the past and the future of a trajectory of a point $\left(\left(-\infty,-t_{0}\right) \cdot x \cup\left(t_{0},+\infty\right) \cdot x, t_{0}>0\right)$ or an open neighbourhood $\left(\left(-\infty,-t_{0}\right) \cdot U \cup\left(t_{0},+\infty\right) \cdot U, t_{0}>0\right)$, we respectively consider the externologies $\varepsilon^{\left(\mathbf{c}, \mathbf{d}_{X}\right)}(X), \varepsilon^{\left(\mathbf{c}, \mathbf{t}_{X}\right)}(X)$.

For the dynamical notions we use the definitions and notations given in the Bhatia and Szego book [4], taking into account that we are working on topological spaces instead of metric spaces and, in some cases, one has to replace sequences by nets. For the notion of attractor used in our study we do not assume the compactness condition considered in [4]. In this paper we have also introduced the dynamic notion of $(\epsilon, \tau)$-agglomerative point (see Definition 6.5) and a notion of basin (see Definition 5.3) which are not contained in [4].

The final section "Conclusions and further work" contains a list with the main relationships (analized in this paper) between exterior and dynamic notions. We also suggest some future research lines: (1) the higher homotopy 
invariant of exterior spaces (Steenrod, Čech and Brown-Grossmann groups) could be very useful tools in the analysis of local and global stability properties of continuous flows and in the study of chaotic dynamical systems; (2) we think that the externologies $\varepsilon^{\left(\epsilon, \tau_{\text {int }}\right)}(X)$ will play an important role in the study of repellers and atractors of a dynamical system, where $\tau_{\text {int }}$ is the intrinsic topology of a flow $X$.

\section{PRELIMINARIES ON EXTERIOR SPACES AND DYNAMICAL SYSTEMS}

2.1. Exterior spaces: End Spaces and Limit Spaces. A continuous map $f: X \rightarrow Y$ is said to be proper if for every closed compact subset $K$ of $Y$, $f^{-1}(K)$ is a compact subset of $X$. The category of topological spaces and the subcategory of spaces and proper maps will be denoted by Top and $\mathbf{P}$, respectively. This last category and its corresponding proper homotopy category are very useful for the study of non-compact spaces and manifolds (see [30]). Nevertheless, one has the problem that $\mathbf{P}$ does not have enough limits and colimits and therefore it is not possible to develop the usual homotopy constructions such as loops, homotopy limits and colimits, et cetera. An answer to this problem is given by the notion of exterior space. The new category of exterior spaces and maps is complete and cocomplete and contains as a full subcategory the category of spaces and proper maps, see $[16,17]$. We refer to $[9,11,12,21,22]$ for further properties and applications of exterior homotopy, and to $[26]$ for a survey of proper homotopy.

Definition 2.1. Let $\left(X, \mathbf{t}_{X}\right)$ be a topological space, where $X$ is the underlying set and $\mathbf{t}_{X}$ its topology. An externology on $\left(X, \mathbf{t}_{X}\right)$ is a non-empty collection $\varepsilon(X)$ of open subsets which is closed under finite intersections and such that if $E \in \varepsilon(X), U \in \mathbf{t}_{X}$ and $E \subset U$ then $U \in \varepsilon(X)$. The members of $\varepsilon(X)$ are called exterior open subsets. An exterior space $\left(X, \varepsilon(X), \mathbf{t}_{X}\right)$ consists of a space $\left(X, \mathbf{t}_{X}\right)$ together with an externology $\varepsilon(X)$. Given an exterior space $\left(X, \varepsilon(X), \mathbf{t}_{X}\right)$ it is useful to work with an exterior basis (or just a basis of the externology), which is nothing else than a subcollection $\beta \subset \varepsilon(X)$ such that for every $E \in \varepsilon(X)$ there exists $F \in \beta$ with $F \subset E$. A map $f:\left(X, \varepsilon(X), \mathbf{t}_{X}\right) \rightarrow\left(X^{\prime}, \varepsilon\left(X^{\prime}\right), \mathbf{t}_{X^{\prime}}\right)$ is said to be an exterior map if it is continuous and $f^{-1}(E) \in \varepsilon(X)$, for all $E \in \varepsilon\left(X^{\prime}\right)$.

The category of exterior spaces and maps will be denoted by $\mathbf{E}$. Given a space $\left(X, \mathbf{t}_{X}\right)$, we can always consider the trivial exterior space taking $\varepsilon=\{X\}$ or the total exterior space if one takes $\varepsilon=\mathbf{t}_{X}$. An important example of externology on a given topological space $X$ is the one constituted by the complements of all closed-compact subsets of $X$. It will be called the cocompact externology and usually written as $\varepsilon^{\mathbf{c}}(X)$. The category of spaces and proper maps can be considered as a full subcategory of the category of exterior spaces via the full embedding $(\cdot)^{\mathbf{c}}: \mathbf{P} \hookrightarrow \mathbf{E}$. The functor $(\cdot)^{\mathbf{c}}$ carries a space $X$ to the exterior space $X^{\mathbf{c}}$ which is endowed with the topology 
of $X$ and the externology $\varepsilon^{\mathbf{c}}(X)$. A map $f: X \rightarrow Y$ is carried to the exterior map $f^{\mathbf{c}}: X^{\mathbf{c}} \rightarrow Y^{\mathbf{c}}$ given by $f^{\mathbf{c}}=f$. It is easy to check that a continuous map $f: X \rightarrow Y$ is proper if and only if $f=f^{\mathbf{c}}: X^{\mathbf{c}} \rightarrow Y^{\mathbf{c}}$ is exterior.

An important role in this paper will be played by the following construction: Let $\left(X, \varepsilon(X), \mathbf{t}_{X}\right)$ be an exterior space, and let $\left(Y, \mathbf{t}_{Y}, \tau\right)$ be a set $Y$ endowed with two topologies $\mathbf{t}_{Y}, \tau$ such that $\mathbf{t}_{Y} \subset \tau$. For $y \in Y$ we denote by $\tau_{y}$ the family of open neighbourhoods of $(Y, \tau)$ at $y$. Given the topological spaces $\left(X, \mathbf{t}_{X}\right),\left(Y, \mathbf{t}_{Y}\right)$, we consider on $X \times Y$ the product topology which is denoted by $\mathbf{t}_{X \times Y}$ and the externology $\varepsilon\left(X \overline{\times}_{\tau} Y\right)$ given by those $E \in \mathbf{t}_{X \times Y}$ such that for each $y \in Y$ there exists $U_{y} \in \tau_{y}$ and $T^{y} \in \varepsilon(X)$ such that $T^{y} \times U_{y} \subset E$. The resulting exterior space $\left(X \times Y, \varepsilon\left(X \overline{\times}_{\tau} Y\right), \mathbf{t}_{X \times Y}\right)$ will be denoted by $X \overline{\times}_{\tau} Y$.

In some contexts, for instance working with exterior homotopies, it is frequent to take $\tau=\mathbf{t}_{Y}$. For these cases, we will use the shorter notation

$$
X \overline{\times} Y=\left(X \times Y, \varepsilon\left(X \overline{\times}_{\mathbf{t}_{Y}} Y\right), \mathbf{t}_{X \times Y}\right)=X \overline{\times}_{\mathbf{t}_{Y}} Y .
$$

Therefore, for these cases we have an induced functor:

$$
(\cdot) \overline{\times}(\cdot): \mathbf{E} \times \mathbf{T o p} \rightarrow \mathbf{E},(X, Y) \rightarrow X \overline{\times} Y .
$$

When $Y$ is a compact space, we have that $E$ is an exterior open subset of $X \overline{\times} Y$ if and only if it is an open subset and there exists $G \in \varepsilon(X)$ such that $G \times Y \subset E$. Furthermore, if $Y$ is a compact space and $\varepsilon(X)=\varepsilon^{\mathbf{c}}(X)$, then $\varepsilon(X \overline{\times} Y)$ coincides with $\varepsilon^{\mathbf{c}}(X \times Y)$ the externology of the complements of closed-compact subsets of $X \times Y$. We also note that if $Y$ is a discrete space, then $E$ is an exterior open subset of $X \overline{\times} Y$ if and only if it is open and for each $y \in Y$ there is $T^{y} \in \varepsilon(X)$ such that $T^{y} \times\{y\} \subset E$.

This bar construction $(\bar{x})$ provides a natural way to define exterior homotopy in E. Indeed, if $I$ denotes the usual closed unit interval $[0,1]$, given exterior maps $f, g: X \rightarrow Y$, it is said that $f$ is exterior homotopic to $g$ if there exists an exterior map $H: X \overline{\times} I \rightarrow Y$ (called exterior homotopy) such that $H(x, 0)=f(x)$ and $H(x, 1)=g(x)$, for all $x \in X$. The corresponding homotopy category of exterior spaces will be denoted by $\pi \mathbf{E}$. Similarly, the usual homotopy category of topological spaces will be denoted by $\pi$ Top.

In this subsection we also deal with special limit constructions associated to any exterior space. In particular we recall the functors $L, \bar{L}, \check{\pi}_{0}, \check{\pi}_{0}: \mathbf{E} \rightarrow$ Top introduced in [19].

Given an exterior space $X=(X, \varepsilon(X))$, its externology $\varepsilon(X)$ can be considered as an inverse system of spaces $\{E\}_{E \in \varepsilon(X)}$. Recall that an inverse system of topological spaces is a functor $Z: I \rightarrow$ Top, where $I$ is a directed set. The functor $Z$ carries $i \geq j, i, j \in I$, to $Z_{j}^{i}: Z_{i} \rightarrow Z_{j}$. The inverse limit of $Z=\left\{Z_{i}\right\}$ is denoted by $\lim _{i \in I} Z_{i}$ (or just by $\lim Z_{i}$ ). An element of the inverse limit can be represented by an element $\left(z_{i}\right)_{i \in I}$ of the product $\prod_{i \in I} Z_{i}$ 
satisfying that $Z_{j}^{i}\left(z_{i}\right)=z_{j}, i \geq j$. For more results and properties about inverse systems, we refer the reader to [10].

For a topological space $Y, \pi_{0}(Y)$ denotes the set of path-components of $Y$ and we have a canonical map $Y \rightarrow \pi_{0}(Y)$ which induces a quotient topology on $\pi_{0}(Y)$. We remark that if $Y$ is locally path-connected, then $\pi_{0}(Y)$ is a discrete space. We also recall that given a subset $A$ of a topological space $Y$ the closure of $A$ is denoted by $\bar{A}$.

Given an exterior space $X=(X, \varepsilon(X))$, for the inverse systems

$\varepsilon(X)=\{E\}_{E \in \varepsilon(X)}, \quad \pi_{0} \varepsilon(X)=\left\{\pi_{0}(E)\right\}_{E \in \varepsilon(X)}, \quad \bar{\pi}_{0} \varepsilon(X)=\left\{\pi_{0}(\bar{E})\right\}_{E \in \varepsilon(X)}$,

we can take inverse limits to obtain the following notions:

Definition 2.2. Let $X=(X, \varepsilon(X))$ be an exterior space. The limit space of $X$ is the topological subspace:

$$
L(X)=\lim \varepsilon(X)=\cap_{E \in \varepsilon(X)} E .
$$

The end space of $X$ is the inverse limit:

$$
\check{\pi}_{0}(X)=\lim \pi_{0} \varepsilon(X)=\lim _{E \in \varepsilon(X)} \pi_{0}(E)
$$

endowed with the inverse limit topology of the spaces $\pi_{0}(E)$.

The bar-limit space of $X$ is the topological subspace:

$$
\bar{L}(X)=\lim \left(\{\bar{E}\}_{E \in \varepsilon(X)}\right)=\cap_{E \in \varepsilon(X)} \bar{E} .
$$

The bar-end space of $X$ is the inverse limit:

$$
\check{\pi}_{0}(X)=\lim \bar{\pi}_{0} \varepsilon(X)=\lim _{E \in \varepsilon(X)} \pi_{0}(\bar{E})
$$

endowed with the inverse limit topology of the spaces $\pi_{0}(\bar{E})$.

It is interesting to observe that if $X$ is an exterior space and $X$ is locally path-connected, then $\check{\pi}_{0}(X)$ is a prodiscrete space.

We note that $\check{\pi}_{0}(X)$ and $\check{\pi}_{0}(X)$ are generalizations to exterior spaces of the space of Freudenthal end points of a topological space. Indeed, if for a topological space $X$ we consider the externology $\varepsilon^{\mathbf{c}}(X)$, then one has that $\check{\pi}_{0}(X)$ is precisely the space of Freudenthal ends of the space $X$, see $[13,23]$. This is the reason why, sometimes, the elements of $\check{\pi}_{0}(X), \check{\bar{\pi}}_{0}(X)$ are also called Freudenthal end points (or Freudenthal ends) of the exterior space $X$. We also mention that the set of Freudenthal end points of an exterior space is a zero dimensional analogue of the Čech invariant of a pro-space (see $[10]$ ) or of the Borsuk invariant (see $[6,7]$ ) given in shape theory.

For every $E \in \varepsilon(X)$, we have a commutative diagram: 


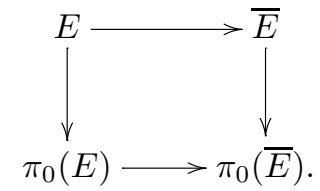

Then, one has the natural transformations of the following commutative diagram:

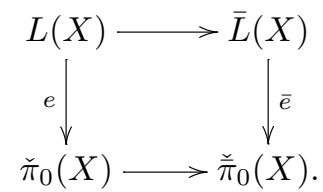

Definition 2.3. Given an exterior space $X=(X, \varepsilon(X))$, an end point $a \in \check{\pi}_{0}(X)$ is said to be e-representable if there is $x \in L(X)$ such that $e(x)=a$. Similarly, a point $b \in \check{\bar{\pi}}_{0}(X)$ is $\bar{e}$-representable if there is $x \in \bar{L}(X)$ such that $\bar{e}(x)=b$.

Notice that the maps $e: L(X) \rightarrow \check{\pi}_{0}(X), \bar{e}: L(X) \rightarrow \check{\bar{\pi}}_{0}(X)$ induce an $e$-decomposition and an $\bar{e}$-decomposition

$$
L(X)=\bigsqcup_{a \in \tilde{\pi}_{0}(X)} L_{a}(X), \quad \bar{L}(X)=\bigsqcup_{b \in \breve{\bar{\pi}}_{0}(X)} \bar{L}_{b}(X)
$$

where $L_{a}(X)=e^{-1}(a)$ and $\bar{L}_{b}(X)=\bar{e}^{-1}(b)$.

It is not difficult to check that the functors $L, \bar{L}$ preserve homotopies and the functors $\check{\pi}_{0}, \check{\pi}_{0}$ are invariant by exterior homotopy. As a consequence of these facts one has:

Proposition 2.4. The functors $L, \bar{L}, \check{\pi}_{0}, \check{\pi}_{0}: \mathbf{E} \rightarrow$ Top induce functors

$$
L, \bar{L}: \pi \mathbf{E} \rightarrow \pi \text { Top, } \quad \check{\pi}_{0}, \check{\pi}_{0}: \pi \mathbf{E} \rightarrow \text { Top. }
$$

REMARK 2.5. A more exhaustive study of these functors and their properties can be seen at $[19,20]$. We can also consider other type of functors:

- Taking the set $c(E)(c(\bar{E}))$ of connected components, for every $E \in$ $\varepsilon(X)$, instead of path-connected components, one has some analogous functors: $\check{c} \check{\bar{c}}: \pi \mathbf{E} \rightarrow$ Top, see [19].

- Some flow-completion functors, related to the Freudenthal compactification of a space (see [13]), are constructed in [20].

2.2. Dynamical Systems, omega limits and prolongational omega limits. Next we recall some elementary concepts about dynamical systems.

Definition 2.6. A dynamical system (or flow) on a topological space $X$ is a continuous map $\varphi: \mathbb{R} \times X \rightarrow X, \varphi(t, x)=t \cdot x$, such that 
(i) $0 \cdot x=x, \forall x \in X$

(ii) $t \cdot(s \cdot x)=(t+s) \cdot x, \forall x \in X, \forall t, s \in \mathbb{R}$.

A flow on $X$ will be denoted by $(X, \varphi)$ and, when no confusion is possible, we use $X$ for short.

For a subset $A \subset X$, we $\operatorname{denote} \operatorname{inv}(A)=\{x \in A \mid \mathbb{R} \cdot x \subset A\}$.

Definition 2.7. A subset $S$ of a flow $X$ is said to be invariant if $\operatorname{inv}(S)=$ $S$.

Given a flow $(X, \varphi)$ one has a subgroup $\left\{\varphi_{t}: X \rightarrow X \mid t \in \mathbb{R}\right\}$ of homeomorphisms, $\varphi_{t}(x)=\varphi(t, x)$, and a family of trajectories (motions, orbits) $\left\{\varphi^{x}: \mathbb{R} \rightarrow X \mid x \in X\right\}, \varphi^{x}(t)=\varphi(t, x)$.

Definition 2.8. Given two flows $(X, \varphi),(Y, \psi)$, a flow morphism $f:(X, \varphi) \rightarrow(Y, \psi)$ is a continuous map $f: X \rightarrow Y$ such that $f(t \cdot x)=t \cdot f(x)$ for every $t \in \mathbb{R}$ and for every $x \in X$.

We note that if $S \subset X$ is invariant, $S$ has a flow structure and the inclusion is a flow morphism. We denote by $\mathbf{F}$ the category of flows and flow morphisms.

Definition 2.9. For a flow $X$, the omega-limit set (in the literature one can find the alternative terminology: $\Lambda^{+}$-limit, right-limit set or positive limit set) of a point $x \in X$ is given as follows:

$$
\Lambda^{+}(x)=\left\{y \in X \mid \exists \text { a net } t_{\delta} \rightarrow+\infty \text { such that } t_{\delta} \cdot x \rightarrow y\right\} .
$$

Note that the subset $\Lambda^{+}(x)$ admits the alternative definition

$$
\Lambda^{+}(x)=\bigcap_{t \geq 0} \overline{[t,+\infty) \cdot x}
$$

which has the advantage of showing that $\Lambda^{+}(x)$ is closed.

Definition 2.10. The $\Lambda^{+}$-limit set of a flow $X$ is the following invariant subset:

$$
\Lambda^{+}(X)=\bigcup_{x \in X} \Lambda^{+}(x) .
$$

Definition 2.11. For a flow $X$, the prolongational omega-limit set (or prolongational right limit set, or prolongational positive limit set) of a point $x \in X$ is given as follows:

$J^{+}(x)=\left\{y \in X \mid \exists\right.$ a net $x_{\delta} \rightarrow x$ and a net $t_{\delta} \rightarrow+\infty$ such that $\left.t_{\delta} \cdot x_{\delta} \rightarrow y\right\}$.

Note that the prolongational omega-limit set admits the alternative definition

$$
J^{+}(x)=\bigcap_{t \geq 0, U \in \mathbf{t}_{X_{x}}} \overline{[t,+\infty) \cdot U}
$$

which has the advantage of showing that $J^{+}(x)$ is closed. 
Definition 2.12. The $J^{+}$-limit set of a flow $X$ is the following invariant subset:

$$
J^{+}(X)=\bigcup_{x \in X} J^{+}(x)
$$

REMARK 2.13. We note that for the set $J^{+}(x)$ Bhatia and Szego ([4]) use the longer expression "first positive prolongational limit set" of $x$.

Now we introduce the basic notions of critical, periodic and Poisson stable points.

Let $X$ be a flow. A point $x \in X$ is said to be a critical point (or fixed point) if for every $t \in \mathbb{R}, t \cdot x=x$. We denote by $C(X)$ the invariant subset of critical points of $X$. A point $x \in X$ is said to be periodic if there is $t \in \mathbb{R}$, $t \neq 0$ such that $t \cdot x=x$. We denote by $P(X)$ the invariant subset of periodic points of $X$.

It is clear that any critical point is a periodic point. Then

$$
C(X) \subset P(X) .
$$

If $x \in X$ is a periodic point but not critical, then there is a real number $t \neq 0$ such that $t \cdot x=x$ and $t$ is called a period of $x$. The smallest positive period $t_{0}$ of $x$ is called the fundamental period of $x$.

A point $x \in X$ is said to be positively Poisson-stable if there is a net $t_{\delta} \rightarrow+\infty$ such that $t_{\delta} \cdot x \rightarrow x$; that is, $x \in \Lambda^{+}(x)$. We will denote by Poisson $^{+}(X)$ the invariant subset of positively Poisson-stable points of $X$.

The reader can easily check that

$$
P(X) \subset \operatorname{Poisson}^{+}(X) \subset \Lambda^{+}(X) .
$$

The notions above can be dualized by considering the ones of alpha-limit sets $\Lambda^{-}(x), J^{-}(x)$ of a point $x$, negatively Poisson-stable points, et cetera. A point $x$ is said to be Poisson-stable if $x$ is positively Poisson-stable and negatively Poisson-stable. To have a more coherent notation, in some cases, we also use $\mathbf{r}$-Poisson-stable instead of positively Poisson-stable and l-Poissonstable instead of negatively Poisson-stable. For a study of the properties of omega-limits, alpha-limits, et cetera, we refer the reader to [4].

REMARK 2.14. Observe that when $X$ satisfies the first axiom of countability (for instance, when $X$ is metrizable) we can consider sequences instead of nets in the definitions above.

Definition 2.15. A point $x \in X$ is said to be a wandering point if there is $U \in \mathbf{t}_{X_{x}}$ and there is $r_{0} \in \mathbb{R}$ such that for every $r \geq r_{0}, \varphi_{r}(U) \cap U=\emptyset$. Denote by $W(X)$ the open subset of wandering points of $X$ and by $N(X)=X \backslash W(X)$ the closed invariant subset of non-wandering points. Hence a point $x$ is nonwandering if for every open neigbourhood $U$ at $x$ and for every $r_{0} \in \mathbb{R}$, there is $r_{1} \geq r_{0}$ such that $\varphi_{r_{1}}(U) \cap U \neq \emptyset$. 
Remark 2.16. The original definition of wandering point was given by G.D. Birkhoff ([5]).

It is easy to check that $x$ is non-wandering if and only if $x \in J^{+}(x)$ if and only if $x \in J^{-}(x)$, see [4]. Therefore, we have:

Proposition 2.17. Let $X$ be a flow, then

$$
N(X) \subset J^{-}(X) \cap J^{+}(X) \text {. }
$$

We use the notion of region of weak attraction and attraction given in [4], but we remove the compactness condition:

Definition 2.18. Let $X$ be a flow and let $S \subset X$. The region $P A(S)$ of pseudo-attraction of $S$ is defined by

$$
P A(S)=\left\{x \in X \mid \Lambda^{+}(x) \subset S\right\} .
$$

The region $W A(S)$ of weak-attraction of $S$ is defined by

$$
W A(S)=\left\{x \in X \mid \Lambda^{+}(x) \cap S \neq \emptyset\right\} .
$$

The region $A(S)$ of attraction of $S$ is defined by

$$
A(S)=\left\{x \in X \mid \Lambda^{+}(x) \subset S, \Lambda^{+}(x) \neq \emptyset\right\} .
$$

Obviously, $A(S) \subset P A(S) \cap W A(S)$.

Definition 2.19. Let $X$ be a flow and let $S \subset T \subset X$. It is said that $S$ is an attractor of $T$ if $T$ is a neighbourhood of $S$ and $T \subset A(S)$ and $S$ is said to be an attractor if $A(S)$ is a neighbourhood of $S$.

In a dual way, using $\Lambda^{-}(x)$ one has the corresponding repelling notions.

\section{Exterior Dynamical Systems}

Given a flow $(X, \varphi)$, a point $x \in X$ and an open neighbourhood $U \in \mathbf{t}_{X_{x}}$, we want to analyse the following questions: On the one hand, the study of the limits $\Lambda^{-}(x), \Lambda^{+}(x)$ and the prolongational limits $J^{-}(x), J^{+}(x)$ gives an 'end panoramic' of the trajectories of $x$ and neighbourhood points $y \in U$ looking backwards or forwards. On the other hand, the relative position of the points $\varphi_{t}(x)$ and $\varphi_{t}(y)$ when $t \rightarrow-\infty$ or $t \rightarrow+\infty$ with respect to some criterion (distance in a metric space, convergence to the same Freudenthal end, et cetera) induces different stability or sensitivity notions with respect to the initial position $(t=0)$ of the trajectories. All these properties can be analysed by using the constructions and results that have been developed for exterior spaces. The idea is quite simple: A flow is given by a continuous map $\varphi: \mathbb{R} \times X \rightarrow X$, and we can consider appropriate externologies on $\mathbb{R} \times X$ and on $X$ such that $\varphi: \mathbb{R} \times X \rightarrow X$ becomes an exterior map. Using different externologies on $\mathbb{R} \times X$ we can study different dynamical properties related to recurrence and omega limits of a dynamical system. In order to consider 
externologies on $\mathbb{R} \times X$, we take an externology $\epsilon$ on $\mathbb{R}$ and a topology $\tau$ in $X$ such that $\tau$ is finer that the topology $\mathbf{t}_{X}$. In this work, we consider three externologies on $\mathbb{R}$, taking open neighbourhoods at $-\infty$, open neighbourhoods at $+\infty$ and the cocompact externology. Regarding $\tau$, we take $\tau$ the discrete topology $\mathbf{d}_{X}$ or $\tau=\mathbf{t}_{X}$. However, many of the properties developed in this work remain for more general externologies $\epsilon$ on $\mathbb{R}$ and topologies $\tau$ on $X$.

3.1. The category of $(\epsilon, \tau)$-exterior flows. In order to compare exterior spaces with flows we introduce the notion of exterior flow, which is a hybrid containing both the notions of an exterior space and of a flow. We use the convention that the first time that a concept related to an exterior flow is introduced is typed in bold italics.

We consider three externologies on $\mathbb{R}$ which are determined by the following exterior basis (see Definition 2.1):

A basis for $\mathbf{l}$ is

$$
\mathcal{B}(\mathbf{l})=\{(-\infty,-n) \mid n \in \mathbb{N}\} .
$$

$\mathbf{l}=\{U \mid U$ is open and there is $n \in \mathbb{N}$ such that $(-\infty,-n) \subset U\}$.

A basis for $\mathbf{r}$ is

$$
\mathcal{B}(\mathbf{r})=\{(n,+\infty) \mid n \in \mathbb{N}\} .
$$

$\mathbf{r}=\{U \mid U$ is open and there is $n \in \mathbb{N}$ such that $(n,+\infty) \subset U\}$.

A basis for $\mathbf{c}=\varepsilon^{\mathbf{c}}$ is

$$
\mathcal{B}(\mathbf{c})=\{\mathbb{R} \backslash[-n, n] \mid n \in \mathbb{N}\} .
$$

$\mathbf{c}=\{U \mid U$ is open and there is $n \in \mathbb{N}$ such that $\mathbb{R} \backslash[-n, n] \subset U\}$.

We denote the corresponding exterior spaces by $\mathbb{R}^{\mathbf{l}}, \mathbb{R}^{\mathbf{r}}, \mathbb{R}^{\mathbf{c}}$, respectively. We note that in these three cases all the canonical translations $\left(t \rightarrow t+t_{0}\right)$ on $\mathbb{R}$ are exterior maps.

Let $\epsilon$ be an externology on $\mathbb{R}$ such that $\epsilon \in\{\mathbf{l}, \mathbf{r}, \mathbf{c}\}$ and for a topological space $X=\left(X, \mathbf{t}_{X}\right)$ consider a topology $\tau \in\left\{\mathbf{d}_{X}, \mathbf{t}_{X}\right\}$, where $\mathbf{d}_{X}$ is the discrete topology on $X$. Recall that using the procedure given in subsection 2.1, the pair $(\epsilon, \tau)$ induces a canonical externology $\varepsilon^{(\epsilon, \tau)}(\mathbb{R} \times X)$ on the product topological space $\mathbb{R} \times X$ as follows:

$N \in \varepsilon^{(\epsilon, \tau)}(\mathbb{R} \times X)$ if $N$ is an open subset of the product space $\mathbb{R} \times X$ and for every $x \in X$ there is $T^{x} \in \epsilon$ and $U_{x} \in \tau_{x}$ such that $T^{x} \times U_{x} \subset N$.

We note that

$$
\varepsilon^{(\mathbf{c}, \tau)}(\mathbb{R} \times X)=\varepsilon^{(\mathbf{l}, \tau)}(\mathbb{R} \times X) \cap \varepsilon^{(\mathbf{r}, \tau)}(\mathbb{R} \times X)
$$

and $\varepsilon^{(\epsilon, \mathbf{t})}(\mathbb{R} \times X) \subset \varepsilon^{(\epsilon, \mathbf{d})}(\mathbb{R} \times X)$.

Recall that $\mathbb{R}^{\epsilon} \overline{\times}_{\tau} X$ denotes the exterior space $\left(\mathbb{R} \times X, \varepsilon^{(\epsilon, \tau)}(\mathbb{R} \times X)\right)$ and in case that $\tau=\mathbf{t}_{X}$ the notation can be reduced to $\mathbb{R}^{\epsilon} \overline{\times} X$ (see subsection $2.1)$. 
Definition 3.1. Let $X=\left(X, \varepsilon(X), \mathbf{t}_{X}\right)$ be an exterior space and suppose that $(\epsilon, \tau) \in\{\mathbf{l}, \mathbf{r}, \mathbf{c}\} \times\left\{\mathbf{d}_{X}, \mathbf{t}_{X}\right\}$. An $(\epsilon, \tau)$-exterior flow is a continuous flow $\varphi: \mathbb{R} \times X \rightarrow X$ such that

(i) $\varphi: \mathbb{R}^{\epsilon} \bar{X}_{\tau} X \rightarrow X$ is exterior and,

(ii) for any $t \in \mathbb{R}, F_{t}: X \overline{\times} I \rightarrow X, F_{t}(x, s)=\varphi(t s, x), s \in I, x \in X$, is also exterior.

An $(\epsilon, \tau)$-exterior flow morphism of $(\epsilon, \tau)$-exterior flows $f: M \rightarrow N$ is a flow morphism such that $f$ is exterior.

Denote by $\mathbf{E}^{(\epsilon, \tau)} \mathbf{F}$ the category of $(\epsilon, \tau)$-exterior flows and $(\epsilon, \tau)$-exterior flow morphisms. When $\tau=\mathbf{t}_{X}$ or $\tau=\mathbf{d}_{X}$ we have the categories $\mathbf{E}^{(\epsilon, \mathbf{t})} \mathbf{F}$ or $\mathbf{E}^{(\epsilon, \mathbf{d})} \mathbf{F}$, respectively.

REMARK 3.2. Observe that the second statement of the definition above establishes that $F_{t}$ is an exterior homotopy between $\operatorname{id}_{X}$ and $\varphi_{t}: X \rightarrow X$.

The following six cases will be analysed: $(\epsilon, \tau) \in\{\mathbf{l}, \mathbf{r}, \mathbf{c}\} \times\left\{\mathbf{d}_{X}, \mathbf{t}_{X}\right\}$. We note that these six exterior spaces are related by the following diagram

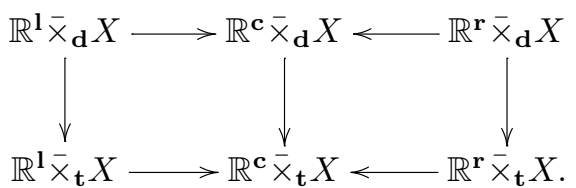

It is worth remarking that this diagram induces the following commutative diagram of forgetful functors:

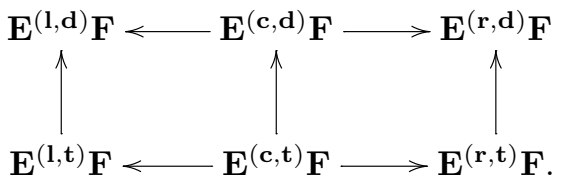

We will use the following notation: if $X=\left(X, \varepsilon(X), \mathbf{t}_{X}\right)$ is an exterior space, then the underlying topological space $\left(X, \mathbf{t}_{X}\right)$ will be denoted by $X_{\mathbf{t}}$.

Since we are working with a new notion mixing the notions of a flow and an exterior space, we always have the following canonical forgetful functors:

Given an $(\epsilon, \tau)$-exterior flow $X=(X, \phi) \in \mathbf{E}^{(\epsilon, \tau)} \mathbf{F}$, one also has a flow $X_{\mathbf{t}}=\left(X_{\mathbf{t}}, \phi\right) \in \mathbf{F}$. This gives a forgetful functor:

$$
(\cdot)_{\mathbf{t}}: \mathbf{E}^{(\epsilon, \tau)} \mathbf{F} \rightarrow \mathbf{F}, X \rightarrow X_{\mathbf{t}} .
$$

For a given $(\epsilon, \tau)$-exterior flow $X=(X, \phi) \in \mathbf{E}^{(\epsilon, \tau)} \mathbf{F}$, we also have that $X$ has the structure of an exterior space with externology $\varepsilon(X)$, then there is a canonical forgetful functor:

$$
(\cdot)^{\varepsilon}: \mathbf{E}^{(\epsilon, \tau)} \mathbf{F} \rightarrow \mathbf{E},(X, \phi) \rightarrow X^{\varepsilon}=(X, \varepsilon(X)) .
$$


This allows us to apply many constructions and properties of exterior spaces to $(\epsilon, \tau)$-exterior flows.

3.2. Exterior flows associated with a flow. The forgetful functor

$$
(\cdot)_{\mathbf{t}}: \mathbf{E}^{(\epsilon, \tau)} \mathbf{F} \rightarrow \mathbf{F}
$$

has a left adjoint that can be constructed as follows:

Given a flow $\varphi: \mathbb{R} \times X \rightarrow X$, an open $N \in \mathbf{t}_{X}$ is said to be $(\epsilon, \tau)$-exterior if for any $x \in X$ there are $T^{x} \in \epsilon$ and $U_{x} \in \tau_{x}$ such that $\varphi\left(T^{x} \times U_{x}\right) \subset N$.

It is easy to check that the family of $(\epsilon, \tau)$-exterior subsets is an externology, that will be denoted by $\varepsilon^{(\epsilon, \tau)}(X)$, and $\varphi$ is an $(\epsilon, \tau)$-exterior flow for the exterior space $X^{(\epsilon, \tau)}=\left(X, \varepsilon^{(\epsilon, \tau)}(X)\right)$.

We also note that, given a flow $X$, one has that $\varepsilon^{(\epsilon, \mathbf{t})}(X) \subset \varepsilon^{(\epsilon, \mathbf{d})}(X)$ and $\varepsilon^{(\mathbf{c}, \tau)}(X)=\varepsilon^{(\mathbf{l}, \tau)}(X) \cap \varepsilon^{(\mathbf{r}, \tau)}(X)$.

Definition 3.3. Given a flow $(X, \varphi)$ and $(\epsilon, \tau) \in\{\mathbf{l}, \mathbf{r}, \mathbf{c}\} \times\left\{\mathbf{d}_{X}, \mathbf{t}_{X}\right\}$, the pair $\left(X^{(\epsilon, \tau)}, \varphi\right)$ is said to be the $(\epsilon, \tau)$-exterior flow induced by $(X, \varphi)$.

When there is no possibility of confusion, $\left(X^{(\epsilon, \tau)}, \varphi\right)$ will be briefly denoted by $X^{(\epsilon, \tau)}$. It is easy to check that $\varepsilon^{(\epsilon, \tau)}(X)$ is the finest externology on $X$ that makes exterior the continuous map $\varphi: \mathbb{R}^{\epsilon} \bar{x}_{\tau} X \rightarrow X$. We also note that if $(X, \varepsilon(X), \varphi)$ is an $(\epsilon, \tau)$-exterior flow, one has that $\varepsilon(X) \subset \varepsilon^{(\epsilon, \tau)}(X)$.

The functor

$$
(\cdot)^{(\epsilon, \tau)}: \mathbf{F} \rightarrow \mathbf{E}^{(\epsilon, \tau)} \mathbf{F}
$$

which carries a flow $X$ to the $(\epsilon, \tau)$-exterior flow $X^{(\epsilon, \tau)}$, permits us to apply to any flow all the constructions developed for exterior flows.

TheOrem 3.4. The functor $(\cdot)^{(\epsilon, \tau)}: \mathbf{F} \rightarrow \mathbf{E}^{(\epsilon, \tau)} \mathbf{F}$ is left adjoint to the functor $(\cdot)_{\mathbf{t}}: \mathbf{E}^{(\epsilon, \tau)} \mathbf{F} \rightarrow \mathbf{F}$. Moreover $(\cdot)_{\mathbf{t}}(\cdot)^{(\epsilon, \tau)}=\mathrm{id}$ and $\mathbf{F}$ can be considered as a full subcategory of $\mathbf{E}^{(\epsilon, \tau)} \mathbf{F}$ via $(\cdot)^{(\epsilon, \tau)}$.

Proof. Let $X$ be in $\mathbf{F}$ and $Y$ be in $\mathbf{E}^{(\epsilon, \tau)} \mathbf{F}$. If $f: X^{(\epsilon, \tau)} \rightarrow Y$ is a morphism in $\mathbf{E}^{(\epsilon, \tau)} \mathbf{F}$, then it is clear that $f=f_{\mathbf{t}}: X=\left(X^{(\epsilon, \tau)}\right)_{\mathbf{t}} \rightarrow Y_{\mathbf{t}}$ is a morphism in $\mathbf{F}$. Now if $g: X \rightarrow Y_{\mathbf{t}}$ is a morphism in $\mathbf{F}$, suppose that $E \in \varepsilon(Y)$. Given $x \in X$, since $Y$ is an $(\epsilon, \tau)$-exterior flow, there are $T^{g(x)} \in \epsilon$ and $V_{g(x)} \in \tau_{Y_{g(x)}}$ such that $T^{g(x)} \cdot V_{g(x)} \subset E$. Since $g: X \rightarrow Y_{\mathbf{t}}$ is continuous, it follows that $g^{-1}\left(V_{g(x)}\right) \in \tau_{X_{x}}$ and $T^{g(x)} \cdot g^{-1}\left(V_{g(x)}\right) \subset g^{-1}(E)$. Therefore $g^{-1}(E) \in \varepsilon^{(\epsilon, \tau)}(X)$. So $g: X^{(\epsilon, \tau)} \rightarrow Y$ is $(\epsilon, \tau)$-exterior and $g_{\mathbf{t}}=g$.

\section{OMEGA-LIMITS OF EXTERIOR FLOWS}

We consider the following notion of omega-limit for exterior flows:

Given a net $t_{\delta}$ in $\mathbb{R}$, if $\epsilon \in\{\mathbf{l}, \mathbf{c}, \mathbf{r}\}$ we say that $t_{\delta}$ is $\epsilon$-divergent if for every $T \in \epsilon$ there is $\delta_{T}$ such that $t_{\delta} \in T$ for every $\delta \geq \delta_{T}$. Note that $t_{\delta}$ is l-divergent if and only if $t_{\delta} \rightarrow-\infty, t_{\delta}$ is c-divergent if and only if $t_{\delta} \rightarrow \infty$ (in the Alexandroff compactification) and $t_{\delta}$ is $\mathbf{r}$-divergent if and only if $t_{\delta} \rightarrow+\infty$. 
Definition 4.1. Let $X$ be an $(\epsilon, \tau)$-exterior flow. Given $x \in X$, the $(\epsilon, \tau)$-omega limit of $x$ is the invariant subspace

$$
\Omega^{(\epsilon, \tau)}(x)=\bigcap_{T \in \epsilon, U \in \tau_{x}} \overline{T \cdot U}
$$

Notice that $y \in \Omega^{(\epsilon, \tau)}(x)$ if and only if there are nets $t_{\delta}$ in $\mathbb{R}$ and $x_{\delta}$ in $X$ such that $t_{\delta}$ is $\epsilon$-divergent, $x_{\delta} \rightarrow x$ in $X_{\tau}$ and $t_{\delta} \cdot x_{\delta} \rightarrow y$ in $X_{\mathbf{t}}$.

The $(\epsilon, \tau)$-omega limit of a subspace $S \subset X$ is the following invariant subset:

$$
\Omega^{(\epsilon, \tau)}(S)=\bigcup_{x \in S} \Omega^{(\epsilon, \tau)}(x)
$$

Notice that we have the following particular cases of omega-limits:

- $\Omega^{(\mathbf{1}, \mathbf{d})}(x)=\Lambda^{-}(x), \quad \Omega^{(1, \mathbf{t})}(x)=J^{-}(x)$;

- $\Omega^{(\mathbf{r}, \mathbf{d})}(x)=\Lambda^{+}(x), \quad \Omega^{(\mathbf{r}, \mathbf{t})}(x)=J^{+}(x)$;

- $\Omega^{(\mathbf{c}, \mathbf{d})}(x)=\Lambda^{-}(x) \cup \Lambda^{+}(x), \quad \Omega^{(\mathbf{c}, \mathbf{t})}(x)=J^{-}(x) \cup J^{+}(x)$.

It is interesting to note that if we change $\Lambda^{+}(x)$ by $\Omega^{(\epsilon, \tau)}(x)$ in Definition 2.18 of region of (pseudo, weak) attraction, we can introduce the notion of region of $(\epsilon, \tau)$-(pseudo, weak) attraction $A^{(\epsilon, \tau)}(S)$ of $S \subset X,\left(P A^{(\epsilon, \tau)}(S)\right.$, $\left.W A^{(\epsilon, \tau)}(S)\right)$.

The use of the notation $\Omega^{(\epsilon, \tau)}(x)$ permits us to unify some common properties of omega-limits, prolongational limits, attractors and repellers (see subsection 2.2). For example, for $S \subset X$, the region of $(\epsilon, \tau)$-attraction of $S$ is given by

$$
A^{(\epsilon, \tau)}(S)=\left\{x \in X \mid \emptyset \neq \Omega^{(\epsilon, \tau)}(x) \subset S\right\} .
$$

In this case, one has that $A^{(\mathbf{r}, \mathbf{d})}(S)=A(S)$ and $A^{(\mathbf{l}, \mathbf{d})}(S)$ is the region of repulsion of $S$.

\section{Limit SPACES, END SPACES AND BASINS OF AN EXTERIOR FLOW}

5.1. Limit spaces and end spaces of an exterior flow. Using the forgetful functor

$$
(\cdot)^{\varepsilon}: \mathbf{E}^{(\epsilon, \tau)} \mathbf{F} \rightarrow \mathbf{E}
$$

and the functors $L, \bar{L}, \check{\pi}_{0}, \check{\pi}_{0}: \mathbf{E} \rightarrow$ Top we have the corresponding composites that will be equipped with the same notation:

$$
L, \bar{L}, \check{\pi}_{0}, \check{\pi}_{0}: \mathbf{E}^{(\epsilon, \tau)} \mathbf{F} \rightarrow \text { Top. }
$$

Proposition 5.1. If $X$ is an $(\epsilon, \tau)$-exterior flow, then:

(i) The spaces $L(X), \bar{L}(X)$ are invariant;

(ii) There are trivial induced flows on $\check{\pi}_{0}(X)$ and $\check{\bar{\pi}}_{0}(X)$. 
Proof. (i) We have that $L(X)=\cap_{E \in \varepsilon(X)} E$ and $\bar{L}(X)=\cap_{E \in \varepsilon(X)} \bar{E}$. It is easy to check that for any $r \in \mathbb{R}, \varphi_{r}(E) \in \varepsilon(X)$ if and only if $E \in \varepsilon(X)$. Therefore

$$
\varphi_{r}(L(X))=\varphi_{r}\left(\cap_{E \in \varepsilon(X)} E\right)=\cap_{E \in \varepsilon(X)} \varphi_{r}(E)=\cap_{E \in \varepsilon(X)} E=L(X) .
$$

Now taking into account that $\overline{\varphi_{r}(E)}=\varphi_{r}(\bar{E})$, a similar result is obtained for $\bar{L}(X)$.

(ii) For each $r \in \mathbb{R}$, consider the exterior homotopy $F_{r}: X \overline{\times} I \rightarrow X$, $F_{r}(x, t)=\varphi(r t, x)$, from id $x_{X}$ to $\varphi_{r}$. By Proposition 2.4, it follows that id $=$ $\check{\pi}_{0}\left(\varphi_{r}\right)$ and similarly id $=\check{\bar{\pi}}_{0}\left(\varphi_{r}\right)$. Therefore the induced actions are trivial.

As a consequence of Proposition 5.1, one also has the corresponding canonical functors

$$
L, \bar{L}, \check{\pi}_{0}, \check{\bar{\pi}}_{0}: \mathbf{E}^{(\epsilon, \tau)} \mathbf{F} \rightarrow \mathbf{F} .
$$

5.2. The beginning and end of a trajectory. This subsection is devoted to the functor $\check{\pi}_{0}$. However similar notions and results are obtained for the functor $\check{\bar{\pi}}_{0}$. We leave the formulation of the corresponding results to the reader.

Let $X$ be an $(\epsilon, \tau)$-exterior flow and suppose that $X$ is $\tau$-locally pathconnected (that is, $(X, \tau)$ is locally path-connected). Then, given $p \in X$ and $E \in \varepsilon(X)$, there are $T^{p} \in \epsilon$ and $U_{p} \in \tau$ such that $p \in U_{p}, U_{p}$ is pathconnected and $T^{p} \cdot U_{p} \subset E$. If $\epsilon \in\{\mathbf{l}, \mathbf{r}\}$ we can also suppose that $T^{p}$ is pathconnected, so $T^{p} \cdot U_{p}$ is path-connected and there is a unique $\omega_{\epsilon}(p, E)$ pathcomponent of $E$ such that $T^{p} \cdot U_{p} \subset \omega_{\epsilon}(p, E) \subset E$. This gives $\tau$-continuous maps $\omega_{\epsilon}(\cdot, E): X \rightarrow \pi_{0}(E), E \in \varepsilon(X)$. Note that if $E_{1} \subset E_{2}$, we have a commutative diagram:

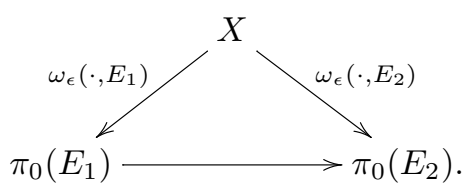

Therefore, by the universal property of the inverse limit, there is an induced map $\omega_{\epsilon}: X \rightarrow \check{\pi}_{0}(X)$ making commutative the following diagram:

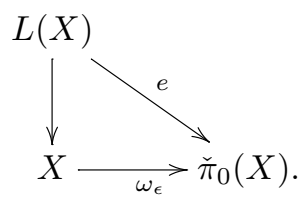

If $\epsilon=\mathbf{c}$, then we can suppose that $T^{p}=T_{1}^{p} \cup T_{\mathbf{r}}^{p}$ has two path-connected components, so that $T^{p} \cdot U_{p}=T_{1}^{p} \cdot U_{p} \cup T_{\mathbf{r}}^{p} \cdot U_{p}$ has two path-connected 
components and there are two unique path-components $\omega_{\mathbf{l}}(p, E), \omega_{\mathbf{r}}(p, E)$ of $E$ such that

$$
T_{1}^{p} \cdot U_{p} \subset \omega_{\mathbf{l}}(p, E) \subset E, \quad T_{\mathbf{r}}^{p} \cdot U_{p} \subset \omega_{\mathbf{r}}(p, E) \subset E .
$$

This gives $\tau$-continuous maps $\omega_{\mathbf{I}}(\cdot, E), \omega_{\mathbf{r}}(\cdot, E): X \rightarrow \pi_{0}(E), E \in \varepsilon(X)$ and induced maps $\omega_{\mathbf{l}}, \omega_{\mathbf{r}}: X \rightarrow \check{\pi}_{0}(X)$ such that the following diagram commutes:

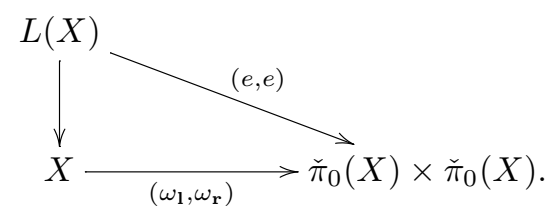

Proposition 5.2. Let $X$ be an $(\epsilon, \tau)$-exterior flow and suppose that $X$ is $\tau$-locally path-connected.

(i) If $\epsilon=1$, then $\omega_{1}: X \rightarrow \check{\pi}_{0}(X)$ is $\tau$-continuous.

(ii) If $\epsilon=\mathbf{r}$, then $\omega_{\mathbf{r}}: X \rightarrow \check{\pi}_{0}(X)$ is $\tau$-continuous.

(iii) If $\epsilon=\mathbf{c}$, then $\left(\omega_{\mathbf{l}}, \omega_{\mathbf{r}}\right): X \rightarrow \check{\pi}_{0}(X) \times \check{\pi}_{0}(X)$ is $\tau$-continuous.

5.3. Basins of an exterior flow.

Definition 5.3. Let $X$ be an $(\epsilon, \tau)$-exterior flow and suppose that $X$ is $\tau$-locally path-connected:

If $\epsilon \in\{\mathbf{l}, \mathbf{c}\}$, denote

$$
X_{(1, b)}=\omega_{1}^{-1}(b), \quad b \in \check{\pi}_{0}(X) .
$$

If $\epsilon \in\{\mathbf{c}, \mathbf{r}\}$ denote

$$
X_{(\mathbf{r}, b)}=\omega_{\mathbf{r}}^{-1}(b), \quad b \in \check{\pi}_{0}(X)
$$

and if $\epsilon=\mathbf{c}$ denote

$$
X_{(a, b)}=\left(\omega_{\mathbf{l}}, \omega_{\mathbf{r}}\right)^{-1}((a, b)), \quad(a, b) \in \check{\pi}_{0}(X) \times \check{\pi}_{0}(X) .
$$

The invariant space $X_{(\mathbf{1}, b)}$ will be called the $\mathbf{l}$-basin at $b, X_{(\mathbf{r}, b)}$ the $\mathbf{r}$ basin at $b$ and $X_{(a, b)}$ the (1, r)-basin at $(a, b)$.

The induced partitions of $X$ in simpler flows

$$
X=\bigsqcup_{b \in \tilde{\pi}_{0}(X)} X_{(\mathbf{l}, b)}, \quad X=\bigsqcup_{b \in \tilde{\pi}_{0}(X)} X_{(\mathbf{r}, b)}, \quad X=\bigsqcup_{(a, b) \in \check{\pi}_{0}(X) \times \check{\pi}_{0}(X)} X_{(a, b)}
$$

will be called $\omega_{\mathbf{l}}$-decomposition, $\omega_{\mathbf{r}}$-decomposition and $\left(\omega_{\mathbf{l}}, \omega_{\mathbf{r}}\right)$-decomposition of the exterior flow $X$, respectively.

Remark 5.4. Since the category of flows can be considered as a full subcategory of this type of categories of exterior flows, the $\omega_{1}$-decomposition

$$
X=\bigsqcup_{b \in \tilde{\pi}_{0}(X)} X_{(1, b)}
$$


can be considered as a generalization for a continuous flow of the decomposition as a disjoint union of "unstable" submanifolds of a differentiable flow. Dually, the $\omega_{\mathbf{r}}$-decomposition divides a flow in "stable" subflows (see $[27-29,31])$.

5.4. Representable, attracting and repelling end points of an exterior flow. Recall that in Definition 2.3 the notion of $e$-representable end point has been introduced for any Freudenthal end of an exterior space. In particular, we also have this notion for exterior flows. Moreover, we can give the following omega-representable notion for end points of exterior flows:

Definition 5.5. Let $X$ be an $(\epsilon, \tau)$-exterior flow and suppose that $X$ is $\tau$-locally path-connected.

If $\epsilon \in\{\mathbf{l}, \mathbf{r}\}$, a Freundenthal end $b \in \check{\pi}_{0}(X)$ is said to be $\omega_{\epsilon}$-representable if $\omega_{\epsilon}^{-1}(b) \neq \emptyset$; and $b$ is said to be $\left(\omega_{\epsilon} \backslash e\right)$-representable if $\omega_{\epsilon}^{-1}(b) \backslash e^{-1}(b) \neq \emptyset$.

If $\epsilon=\mathbf{c}$, given a pair of end points $(a, b) \in \check{\pi}_{0}(X) \times \check{\pi}_{0}(X),(a, b)$ is said to be $\left(\omega_{\mathbf{l}}, \omega_{\mathbf{r}}\right)$-representable if $X_{(a, b)}=\left(\omega_{\mathbf{l}}, \omega_{\mathbf{r}}\right)^{-1}((a, b)) \neq \emptyset$. An end point $a \in \check{\pi}_{0}(X)$ is said to be $\left(\omega_{\mathbf{l}}, \omega_{\mathbf{r}}\right)$-representable if the pair $(a, a)$ is $\left(\omega_{\mathbf{l}}, \omega_{\mathbf{r}}\right)$-representable; finally $a$ is said to be $\left(\left(\omega_{\mathbf{l}}, \omega_{\mathbf{r}}\right) \backslash e\right)$-representable if $\left(\omega_{\mathbf{l}}, \omega_{\mathbf{r}}\right)^{-1}((a, a)) \backslash e^{-1}(a) \neq \emptyset$.

Definition 5.6. Let $X$ be an $(\epsilon, \tau)$-exterior flow and suppose that $X$ is $\tau$-locally path-connected.

If $\epsilon=1$, an end point $b \in \check{\pi}_{0}(X)$ is said to be a repelling end point if $b$ is $\omega_{\mathbf{l}}$-representable and the l-basin $X_{(\mathbf{l}, b)}$ is an open subset.

If $\epsilon=\mathbf{r}$, an end point $b \in \check{\pi}_{0}(X)$ is said to be an attracting end point if $b$ is $\omega_{\mathbf{r}}$-representable and the $\mathbf{r}$-basin $X_{(\mathbf{r}, b)}$ is an open subset.

If $\epsilon=\mathbf{c}$, a pair of end points $(a, b) \in \check{\pi}_{0}(X) \times \check{\pi}_{0}(X)$ is said to be a repelling-attracting pair if $(a, b)$ is $\left(\omega_{\mathbf{l}}, \omega_{\mathbf{r}}\right)$-representable and the $(\mathbf{l}, \mathbf{r})$ basin $X_{(a, b)}$ is an open subset.

5.5. Limit flows, bar-limit flows and Freudenthal-end spaces of a flow. If we consider the functor given in subsection 3.2

$$
(\cdot)^{(\epsilon, \tau)}: \mathbf{F} \rightarrow \mathbf{E}^{(\epsilon, \tau)} \mathbf{F}
$$

and the functors developed in subsection 5.1,

$$
L, \bar{L}, \check{\pi}_{0}, \check{\pi}_{0}: \mathbf{E}^{(\epsilon, \tau)} \mathbf{F} \rightarrow \mathbf{F},
$$

we can consider the composites

$$
L^{(\epsilon, \tau)}=L(\cdot)^{(\epsilon, \tau)}, \bar{L}^{(\epsilon, \tau)}=\bar{L}(\cdot)^{(\epsilon, \tau)}, \check{\pi}_{0}^{(\epsilon, \tau)}=\check{\pi}_{0}(\cdot)^{(\epsilon, \tau)}, \check{\bar{\pi}}_{0}^{(\epsilon, \tau)}=\check{\bar{\pi}}_{0}(\cdot)^{(\epsilon, \tau)}
$$

that will be taken as functors from $\mathbf{F}$ to $\mathbf{F}$. 


\section{Limit FLOW OF AN EXTERIOR FLOW, PERIODIC POINTS AND AGGLOMERATIVE POINTS}

In this section we analyse the relation between exterior closed singletons and periodic and agglomerative points.

6.1. Exterior $(\epsilon, \mathbf{d})$-closed singletons and periodic points. In this subsection, for a point $x$ in a flow $X$ we see that the dynamic property of being a non-periodic point can be reformulated by using exterior terminology by the fact that $X \backslash\{x\}$ is an $(\epsilon, \mathbf{d})$-exterior open subset. Moreover, the subflow of periodic points is interpreted as the limit space of the exterior space $X^{(\epsilon, \mathbf{d})}$.

Lemma 6.1. Let $X$ be an $(\epsilon, \mathbf{d})$-exterior flow and suppose that $x \in X$. If $x$ is a periodic point, then for every $E \in \varepsilon(X), x \in E$. Consequently, $P(X) \subset L(X)$.

Proof. Suppose that $x$ is a periodic point. If $E \in \varepsilon(X)$, then there is $T \in \epsilon$ such that $T \cdot x \subset E$. Since $x$ is periodic, $T \cdot x=\mathbb{R} \cdot x$; taking into account that $x \in \mathbb{R} \cdot x$, we have that that $x \in E$.

Theorem 6.2. Let $X$ be a $T_{1}$ flow. If $x \in X$, then the following statements are equivalent:

(i) $x$ is a non-periodic point,

(ii) $X \backslash\{x\}$ is an $(\epsilon, \mathbf{d})$-exterior subset.

Proof. Suppose that $x$ is a non-periodic point. Take $y$ in $X$. If $y$ is not in the trajectory of $x$, then for every $T \in \epsilon, T \cdot y \subset X \backslash\{x\}$. If $y$ is in the trajectory of $x$, taking into account that $x$ is not periodic, there is $T \in \epsilon$ such that $T \cdot y \subset X \backslash\{x\}$. Then $X \backslash\{x\}$ is $(\epsilon, \mathbf{d})$-exterior.

Conversely, suppose that $x$ is a periodic point. Then, by Lemma 6.1 above, $X \backslash\{x\}$ is not $(\epsilon, \mathbf{d})$-exterior.

TheOREM 6.3. Let $X$ be a $T_{1}$ flow. Then, $L^{(\epsilon, \mathbf{d})}(X)=P(X)$ is precisely the set of periodic points of $X$.

Proof. Let $x \in X \backslash P(X)$. By Theorem $6.2, X \backslash\{x\}$ is an $(\epsilon, \mathbf{d})$-exterior subset and this implies that $x \notin L^{(\epsilon, \mathbf{d})}(X)$. Then, $L^{(\epsilon, \mathbf{d})}(X) \subset P(X)$ and, by Lemma 6.1, we have that $L^{(\epsilon, \mathbf{d})}(X)=P(X)$.

REMARK 6.4. As a consequence of this result, one has that for $T_{1}$ flows $X$ the construction $L^{(\epsilon, \mathbf{d})}$ is idempotent:

$$
L^{(\epsilon, \mathbf{d})} L^{(\epsilon, \mathbf{d})}(X)=L^{(\epsilon, \mathbf{d})}(X) .
$$


6.2. Exterior $(\epsilon, \mathbf{t})$-closed singletons and agglomerative points. Recall that in Definition 2.9 we have considered the omega-limit $\left(\Lambda^{+}\right.$-limit $)$set $\Lambda^{+}(x)$ of a point $x$ in a flow $X$ and after Definition 4.1 we have seen that $\Omega^{(\mathbf{r}, \mathbf{d})}(x)=\Lambda^{+}(x)$.

In this subsection, for a point $x$ in a flow $X$ we see that the dynamic property $\Lambda^{+}(x)=\emptyset$ can be reformulated by using exterior terminology by the fact that $X \backslash\{x\}$ is an $(\mathbf{l}, \mathbf{t})$-exterior open subset. Moreover, the subflow $\left\{x \in X \mid \Lambda^{+}(x) \neq \emptyset\right\}$ is interpreted as the limit space of the exterior space $X^{(1, \mathbf{t})}$.

We consider the following notion of agglomerative point for exterior flows:

Definition 6.5. Let $X$ be an $(\epsilon, \tau)$-exterior flow. Given $x \in X, x$ is said to be an $(\epsilon, \tau)$-agglomerative point if

$$
\Omega^{(\epsilon, \tau)}(x) \neq \emptyset .
$$

We denote by $A g^{(\epsilon, \tau)}(X)$ the invariant subspace of $(\epsilon, \tau)$-agglomerative points of $X$. Note that $A g^{(\epsilon, \tau)}(X)=A^{(\epsilon, \tau)}(X)$ is the region of $(\epsilon, \tau)$-attraction of $X$ (see Definition 4.1). In particular, $A g^{(\mathbf{r}, \mathbf{d})}(X)=A(X)$ is the region of attraction of $X$, see Definition 2.18. If $X$ is a flow, given $x \in X, x$ is said an $(\epsilon, \tau)$-agglomerative point if $x$ is $(\epsilon, \tau)$-agglomerative in $X^{(\epsilon, \tau)} . A g^{(\epsilon, \tau)}(X)$ denotes the subflow of $(\epsilon, \tau)$-agglomerative points of $X$.

Proposition 6.6. Let $X$ be an $(\epsilon, \tau)$-exterior flow where $\epsilon \in\{\mathbf{l}, \mathbf{r}\}$. If $x_{1} \in \Omega^{(\epsilon, \mathbf{d})}\left(x_{0}\right)$ and $x_{2} \in \Omega^{(\epsilon, \mathbf{d})}\left(x_{1}\right)$, then $x_{2} \in \Omega^{(\epsilon, \mathbf{d})}\left(x_{0}\right)$.

Proof. In this proof we suppose that $\epsilon=$ r. Similarly, one has an analogous proof for $\epsilon=1$.

Taking into account that $x_{2} \in \Omega^{(\mathbf{r}, \mathbf{d})}\left(x_{1}\right)$, for each $U \in \mathbf{t}_{X_{x_{2}}}$ and $t \geq 0$ there is $r_{U}^{t} \in \mathbb{R}_{+}$such that $r_{U}^{t} \geq t$ and $r_{U}^{t} \cdot x_{1} \in U$. Since $x_{1} \in \Omega^{(\mathbf{r}, \mathbf{d})}\left(x_{0}\right)$, we also have that for each $V \in \mathbf{t}_{X_{x_{1}}}$ and $s \geq 0$ there is $r_{V}^{s} \in \mathbb{R}_{+}$such that $r_{V}^{s} \geq s$ and $r_{V}^{s} \cdot x_{0} \in V$.

Given $U \in \mathbf{t}_{X_{x_{2}}}$ and $t \geq 0$, since $r_{U}^{t} \cdot x_{1} \in U$ and the flow is continuous, there is $V_{U}^{t} \in \mathbf{t}_{X_{x_{1}}}$ such that $r_{U}^{t} \cdot V_{U}^{t} \subset U$. For $V_{U}^{t} \in \mathbf{t}_{X_{x_{1}}}$ and $s=0$, there are $r_{V_{U}^{t}}^{0} \in \mathbb{R}_{+}$such that $r_{V_{U}^{t}}^{0} \cdot x_{0} \in V_{U}^{t}$. This implies that

$$
\left(r_{U}^{t}+r_{V_{U}^{t}}^{0}\right) \geq t+0=t
$$

and

$$
\left(r_{U}^{t}+r_{V_{U}^{t}}^{0}\right) \cdot x_{0}=r_{U}^{t} \cdot\left(r_{V_{U}^{t}}^{0} \cdot x_{0}\right) \in r_{U}^{t} \cdot V_{U}^{t} \subset U .
$$

Therefore $x_{2} \in \overline{[t,+\infty) \cdot x_{0}}$ for every $t \geq 0$, so $x_{2} \in \Omega^{(\mathbf{r}, \mathbf{d})}\left(x_{0}\right)$.

For the following results we use the notation $-\mathbf{l}=\mathbf{r},-\mathbf{r}=\mathbf{l}$ and $-\mathbf{c}=\mathbf{c}$.

Proposition 6.7. Let $X$ be an $(\epsilon, \mathbf{t})$-exterior flow. If $x_{1} \in \Omega^{(\epsilon, \mathbf{t})}\left(x_{0}\right)$, then $x_{0} \in \Omega^{(-\epsilon, \mathbf{t})}\left(x_{1}\right)$. 
Proof. Suppose that there are nets $x_{\delta}$ and $t_{\delta}$ such that $x_{\delta} \rightarrow x_{0}, t_{\delta}$ is $\epsilon$-divergent and $t_{\delta} \cdot x_{\delta} \rightarrow x_{1}$. Then, we have that $-t_{\delta}$ is $(-\epsilon)$-divergent and $\left(-t_{\delta}\right) \cdot\left(t_{\delta} \cdot x_{\delta}\right) \rightarrow x_{0}$. Therefore $x_{0} \in \Omega^{(-\epsilon, \mathbf{t})}\left(x_{1}\right)$.

Corollary 6.8. Let $X$ be a flow. Then

$$
A g^{(-\epsilon, \mathbf{d})}(X) \subset A g^{(-\epsilon, \mathbf{t})}(X) \subset \Omega^{(\epsilon, \mathbf{t})}(X) .
$$

Proof. Given $x \in X$, we have that $\Omega^{(-\epsilon, \mathbf{d})}(x) \subset \Omega^{(-\epsilon, \mathbf{t})}(x)$. If $\emptyset \neq$ $\Omega^{(-\epsilon, \mathbf{d})}(x)$, then $\emptyset \neq \Omega^{(-\epsilon, \mathbf{t})}(x)$. This implies that

$$
A g^{(-\epsilon, \mathbf{d})}(X) \subset A g^{(-\epsilon, \mathbf{t})}(X) .
$$

Now, take $x \in A g^{(-\epsilon, \mathbf{t})}(X)$, then $\Omega^{(-\epsilon, \mathbf{t})}(x) \neq \emptyset$. Take $y \in \Omega^{(-\epsilon, \mathbf{t})}(x)$, by Proposition 6.7, $x \in \Omega^{(\epsilon, \mathbf{t})}(y) \subset \Omega^{(\epsilon, \mathbf{t})}(X)$. Therefore, $x \in \Omega^{(\epsilon, \mathbf{t})}(X)$.

Theorem 6.9. Let $X$ be a $T_{1}$ flow. Then the following statements hold:

(i) Given $x, y \in X$, there is an open neighbourhood $V_{y}$ at $y$ and there is $T \in \epsilon$ such that $x \notin T \cdot V_{y}$ if and only if $y \notin \Omega^{(-\epsilon, \mathbf{d})}(x)$,

(ii) $X \backslash\{x\}$ is an $(\epsilon, \mathbf{t})$-exterior subset if and only if $\Omega^{(-\epsilon, \mathbf{d})}(x)=\emptyset$.

Proof. (i) Suppose that there is an open neighbourhood $V_{y}$ at $y$ and there is $T \in \epsilon$ such that $x \notin T \cdot V_{y}$. If we assume that $y \in \Omega^{(-\epsilon, \mathbf{d})}(x)$, then there is an $(-\epsilon)$-divergent net $t_{\delta}$ such that $t_{\delta} \cdot x \in V_{y}$ and $-t_{\delta} \in T$ for every $\delta \geq \delta_{0}$. If we denote $v_{\delta_{0}}=t_{\delta_{0}} \cdot x \in V_{y}$, one has that $x=\left(-t_{\delta_{0}}\right) \cdot v_{\delta_{0}} \in T \cdot V_{y}$, which is a contradiction. Therefore, $y \notin \Omega^{(-\epsilon, \mathbf{d})}(x)$.

Conversely, suppose that $y \notin \Omega^{(-\epsilon, \mathbf{d})}(x)$ and for every $V_{y}$ and every $T \in \epsilon$, $x \in T \cdot V_{y}$. Note that

$$
\Delta=\left\{\left(T, V_{y}\right) \mid t \in \epsilon, V_{y} \in \mathbf{t}_{X_{y}}\right\}
$$

is a directed set, $\left.\delta=\left(T, V_{y}\right) \leq \delta^{\prime}=\left(T^{\prime}, V_{y}^{\prime}\right)\right\}$ if $T^{\prime} \subset T$ and $V_{y}^{\prime} \subset V_{y}$. Then there are an $\epsilon$-divergent net $\left(t_{\delta}\right)$ and a net $\left(v_{\delta}\right)$ such that $v_{\delta} \rightarrow y$ and $t_{\delta} \cdot v_{\delta}=x$. Thus the net $\left(-t_{\delta}\right)$ verifies that $\left(-t_{\delta}\right) \cdot x=v_{\delta} \rightarrow y$. Therefore $y \in \Omega^{(-\epsilon, \mathbf{d})}(x)$ which is a contradiction. Consequently, there is an open neigbourhood $V_{y}$ at $y$ and there is $T \in \epsilon$ such that $x \notin T \cdot V_{y}$.

(ii) follows from (i).

Corollary 6.10. If $X$ is a $T_{1}$ flow, then

$$
L^{(\epsilon, \mathbf{t})}(X)=A g^{(-\epsilon, \mathbf{d})}(X) .
$$

Proof. If $\Omega^{(-\epsilon, \mathbf{d})}(x)=\emptyset$, then, by Theorem 6.9 (ii), $X \backslash\{x\}$ is an $(\epsilon, \mathbf{t})$ exterior subset and this implies that $x \notin L^{(\epsilon, \mathbf{t})}(X)$. If $x \notin L^{(\epsilon, \mathbf{t})}(X)$, then there is an $(\epsilon, \mathbf{t})$-open subset $E$ such that $x \notin E$. Then, $E \subset X \backslash\{x\}$. Since $X$ is $T_{1}$, we have that $X \backslash\{x\}$ is an $(\epsilon, \mathbf{t})$-exterior subset. By Theorem 6.9 (ii), it follows that $\Omega^{(-\epsilon, \mathbf{d})}(x)=\emptyset$. 
Corollary 6.11. If $X$ is a $T_{1}$ flow, then

(i) $L^{(\mathbf{l}, \mathbf{t})}(X)=\left\{x \in X \mid \Omega^{(\mathbf{r}, \mathbf{d})}(x) \neq \emptyset\right\}=A g^{(\mathbf{r}, \mathbf{d})}(X)$,

(ii) $L^{(\mathbf{r}, \mathbf{t})}(X)=\left\{x \in X \mid \Omega^{(\mathbf{l}, \mathbf{d})}(x) \neq \emptyset\right\}=A g^{(\mathbf{l}, \mathbf{d})}(X)$,

(iii) $L^{(\mathbf{c}, \mathbf{t})}(X)=L^{(\mathbf{l}, \mathbf{t})}(X) \cup L^{(\mathbf{r}, \mathbf{t})}(X)$.

Corollary 6.12. If $X$ is a $T_{1}$ flow, then

$$
L^{(\epsilon, \mathbf{t})}(X) \subset \Omega^{(\epsilon, \mathbf{t})}(X) .
$$

Proof. It follows from Corollary 6.8 and Corollary 6.10.

Corollary 6.13. Let $X$ be a $T_{1}$ compact flow. Then,

$$
L^{(\mathbf{l}, \mathbf{t})}(X)=L^{(\mathbf{r}, \mathbf{t})}(X)=L^{(\mathbf{c}, \mathbf{t})}(X)=X .
$$

PRoOF. It follows from the property of finite intersection of the compact flow $X$ and Corollary 6.10.

TheOREM 6.14. Let $X$ be a $T_{2}$ flow. Suppose that for every $x \in L^{(\epsilon, \mathbf{t})}(X)$, $\Omega^{(-\epsilon, \mathbf{d})}(x)$ is compact. Then,

(i) For every $x \in L^{(\epsilon, \mathbf{t})}(X), \emptyset \neq \Omega^{(-\epsilon, \mathbf{d})}(x) \subset L^{(\epsilon, \mathbf{t})}(X)$;

(ii) $L^{(\epsilon, \mathbf{t})}\left(L^{(\epsilon, \mathbf{t})}(X)\right)=L^{(\epsilon, \mathbf{t})}(X)$;

(iii) $\Omega^{(-\epsilon, \mathbf{d})}(X) \subset L^{(\epsilon, \mathbf{t})}(X)$ and the region of $(-\epsilon, \mathbf{d})$-attraction of $\Omega^{(-\epsilon, \mathbf{d})}(X)$ is $L^{(\epsilon, \mathbf{t})}(X)$.

Proof. (i) Suppose $x \in L^{(\epsilon, \mathbf{t})}(X)$ and take $y \in \Omega^{(-\epsilon, \mathbf{d})}(x)$. Since $\Omega^{(-\epsilon, \mathbf{d})}(x)$ is a closed compact invariant subset of $X$, one has that

$$
\{\overline{T \cdot y} \mid T \in(-\epsilon)\}
$$

is a family of closed subsets of $\Omega^{(-\epsilon, \mathbf{d})}(x)$ with non-empty intersection $\bigcap_{T \in(-\epsilon)} \overline{T \cdot y}=\Omega^{(-\epsilon, \mathbf{d})}(y)$. Note that $\Omega^{(-\epsilon, \mathbf{d})}(y) \subset \Omega^{(-\epsilon, \mathbf{d})}(x)$. (ii) and (iii) follow from (i) and Corollary 6.10.

Given an exterior space $X=(X, \varepsilon(X)), \mathcal{B}$ is a neighbourhood basis of $\varepsilon(X)$ if for every $E \in \varepsilon(X)$ there is $K \in \mathcal{B}$ such that $K \subset E$ and its interior $\operatorname{Int}(K) \in \varepsilon(X)$.

Definition 6.15. An exterior space $X=(X, \varepsilon(X))$ is said to be locally compact at infinity (or $\varepsilon(X)$ is locally compact at infinity) if $\varepsilon(X)$ has a neighbourhood basis $\mathcal{B}$ such that every $K \in \mathcal{B}$ is a compact subset.

Corollary 6.16. Let $X$ be a $T_{2}$ flow. If $\varepsilon^{(\mathbf{r}, \mathbf{d})}(X)$ is locally compact at infinity, then

(i) $L^{(\mathbf{l}, \mathbf{t})}(X)=X=A(X)$,

(ii) $\Omega^{(\mathbf{r}, \mathbf{d})}(X)$ is an attractor of $X$. 
Proof. Take $x \in X$. Since $X^{(\mathbf{r}, \mathbf{d})}$ is locally compact at infinity, there are a compact subset $K$ of $X$ and $T \in \mathbf{r}$ such that $T \cdot x \subset \operatorname{Int}(K) \subset K$. Note that $K$ is a closed subset because $X$ is $T_{2}$, and therefore $\overline{T \cdot x} \subset K$. Applying the finite intersection property we obtain that $\Omega^{(\mathbf{r}, \mathbf{d})}(x)$ is a non-empty compact subset.

Corollary 6.17. Let $X$ be a $T_{2}$ flow. If $\varepsilon^{(\mathbf{c}, \mathbf{d})}(X)$ is locally compact at infinity, then

(i) $L^{(\mathbf{l}, \mathbf{t})}(X)=X=L^{(\mathbf{r}, \mathbf{t})}(X)$

(ii) $\Omega^{(\mathbf{r}, \mathbf{d})}(X)$ is an attractor of $X$,

(iii) $\Omega^{(\mathbf{l}, \mathbf{d})}(X)$ is a repeller of $X$.

In the following, we use the notation $\mathbb{R}_{+}=[0,+\infty)$ and $\mathbb{R}_{-}=(-\infty, 0]$.

Definition 6.18. Let $X$ be a flow. A point $x \in X$ is said to be positively Lagrange-stable if $\overline{\mathbb{R}_{+} \cdot x}$ is compact, $x$ is said to be negatively Lagrange-stable if $\overline{\mathbb{R}_{-} \cdot x}$ is compact and $x$ is said Lagrange-stable if $x$ is positively and negatively Lagrange-stable (note that in this case the closure of the trajectory is compact). A flow $X$ is said to be positively (resp., negatively) Lagrangestable if for every $x \in X, x$ is positively Lagrange-stable (resp., $x$ is negatively Lagrange-stable). $X$ is said to be Lagrange-stable if $X$ is positively and negatively Lagrange-stable.

Corollary 6.19. Let $X$ be a $T_{2}$ flow. If $X$ is positively Lagrange-stable, then

(i) $L^{(\mathbf{l}, \mathbf{t})}(X)=X$,

(ii) $\Omega^{(\mathbf{r}, \mathbf{d})}(X)$ is an attractor of $X$.

REMARK 6.20. Dually, one has that if $\varepsilon^{(\mathbf{l}, \mathbf{d})}(X)$ is locally compact at infinity or $X$ is negatively Lagrange-stable, then $\Omega^{(\mathbf{r}, \mathbf{d})}(X)$ is a repeller of $X$. We also have that if $\varepsilon^{(\mathbf{c}, \mathbf{d})}(X)$ is locally compact at infinity or $X$ is Lagrangestable, then $\Omega^{(\mathbf{c}, \mathbf{d})}(X)$ is an attractor and a repeller of $X$.

REMARK 6.21. For a Morse function ([25]) $f: X \rightarrow \mathbb{R}$, where $X$ is a compact $T_{2}$ Riemannian manifold, one has that the opposite of the gradient of $f$ induces a flow with a finite number of critical points. In this case, we have that $X$ is locally path-connected and the $(\epsilon, \tau)$-exterior flow $X^{(\epsilon, \tau)}$ is locally compact at infinity. Then, one has that

$$
L^{(\epsilon, \mathbf{d})}(X)=\Omega^{(\epsilon, \mathbf{d})}(X)=P(X)=C(X), \quad L^{(\epsilon, \mathbf{t})}(X)=\Omega^{(\epsilon, \mathbf{t})}=X
$$

and $L^{(\epsilon, \mathbf{d})}(X)$ is an attractor and a repeller of $X$. 


\section{BAR-LIMIT SPACE OF AN EXTERIOR FLOW, OMEGA-LIMITS AND ALMOST} PERIODIC POINTS

7.1. Bar-limit space of an exterior flow and omega-limits. We recall that the omega-limit set $\Lambda^{+}(x)$ and the prolongational omega-limit set $J^{+}(x)$ of a point $x$ in a flow $X$ have been defined in Definition 2.9 and Definition 2.11, respectively, and after Definition 4.1 we have seen that $\Omega^{(\mathbf{r}, \mathbf{d})}(x)=\Lambda^{+}(x)$, $\Omega^{(\mathbf{r}, \mathbf{t})}(x)=J^{+}(x)$. Recall the notation $\Omega^{(\epsilon, \tau)}(X)=\cup_{x \in X} \Omega^{(\epsilon, \tau)}(x)$.

In this subsection we prove that under some topological conditions, the subflow closure of $\Omega^{(\mathbf{r}, \mathbf{d})}(X)$ is the bar-limit space of the exterior space $X^{(\mathbf{r}, \mathbf{d})}$ and similarly the subflow closure of $\Omega^{(\mathbf{r}, \mathbf{t})}(X)$ is the bar-limit space of the exterior space $X^{(\mathbf{r}, \mathbf{t})}$. Analogous results are obtained for

$$
\Omega^{(\mathbf{l . d})}(x)=\Lambda^{-}(x), \quad \Omega^{(\mathbf{l}, \mathbf{t})}(x)=J^{-}(x) .
$$

Then, these relevant subflows can be described as bar-limit spaces of exterior spaces.

LEMma 7.1. If $X$ is an $(\epsilon, \tau)$-exterior flow, then

$$
\Omega^{(\epsilon, \tau)}(X) \subset \bar{L}(X)
$$

Proof. If $E \in \varepsilon(X)$, for every $x \in X$ there exist $T \in \epsilon$ and $U \in \tau_{x}$ such that $T \cdot U \subset E$. Taking the closure, we have $\overline{T \cdot U} \subset \bar{E}$. This implies that $\Omega^{(\epsilon, \tau)}(x) \subset \bar{L}(X)$ for every $x \in X$ and therefore $\Omega^{(\epsilon, \tau)}(X) \subset \bar{L}(X)$.

Proposition 7.2. If $X$ is a flow and $\epsilon \in\{\mathbf{l}, \mathbf{c}, \mathbf{r}\}$, then

(i) $\Omega^{(\epsilon, \mathbf{d})}(x) \subset \Omega^{(\epsilon, \mathbf{t})}(x)$, for every $x \in X$,

(ii) the following diagram is commutative

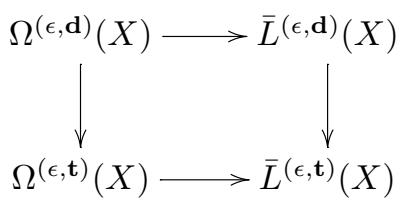

where all the arrows are inclusions.

Proof. (i) It follows from the fact that for any $U \in \tau_{x}$ and $T \in \epsilon$, $T \cdot x \subset T \cdot U$. The rest of the proof is straightforward.

In the following results, it is interesting to take into account that if $X=$ $(X, \varepsilon(X))$ is an $(\epsilon, \tau)$-exterior flow, then $\varepsilon(X) \subset \varepsilon^{(\epsilon, \tau)}\left(X_{\mathbf{t}}\right)$.

Proposition 7.3. Let $X=(X, \varepsilon(X))$ be an $(\epsilon, \tau)$-exterior flow and $x \in$ $X$. Then there exists $V \in\left(\mathbf{t}_{X}\right)_{x}$ such that $X \backslash \bar{V} \in \varepsilon(X)$ if and only if $x \notin \bar{L}(X)$. 
Proof. Suppose that there exists $V \in\left(\mathbf{t}_{X}\right)_{x}$ such that $X \backslash \bar{V} \in \varepsilon(X)$. Since $V \cap(X \backslash \bar{V})=\emptyset$, then $x \notin \overline{X \backslash \bar{V}}$. Since $X \backslash \bar{V} \in \varepsilon(X)$, it follows that

$$
x \notin \bigcap_{E \in \varepsilon(X)} \bar{E}=\bar{L}(X) .
$$

Conversely, if $x \notin \bar{L}(X)$, there is $E \in \varepsilon(X)$ such that $x \notin \bar{E}$; then taking $V=X \backslash \bar{E}=\operatorname{Int}(X \backslash E)$, we have that $V \in\left(\mathbf{t}_{X}\right)_{x}$ and $E \subset X \backslash \bar{V}$, so $X \backslash \bar{V} \in \varepsilon(X)$.

Lemma 7.4. Let $X=(X, \varepsilon(X))$ be an $(\epsilon, \tau)$-exterior flow and $x \in X$. If there exists $V \in\left(\mathbf{t}_{X}\right)_{x}$ such that $X \backslash \bar{V} \in \varepsilon(X)$, then $x \notin \overline{\Omega^{(\epsilon, \tau)}(X)}$.

Proof. Suppose there exists $V \in\left(\mathbf{t}_{X}\right)_{x}$ such that $X \backslash \bar{V} \in \varepsilon(X)$; then by Proposition 7.3, it follows that $x \notin \bar{L}(X)$. By Lemma 7.1, one has that

$$
\Omega^{(\epsilon, \tau)}(X) \subset \overline{\Omega^{(\epsilon, \tau)}(X)} \subset \bar{L}(X),
$$

so $x \notin \overline{\Omega^{(\epsilon, \tau)}(X)}$.

Lemma 7.5. Let $X$ be a locally compact regular flow. If $x \notin \overline{\Omega^{(\epsilon, \tau)}(X)}$, then there exists $V \in\left(\mathbf{t}_{X}\right)_{x}$ such that $X \backslash \bar{V}$ is $(\epsilon, \tau)$-exterior.

Proof. Suppose that $x \notin \overline{\Omega^{(\epsilon, \tau)}(X)}$. Since $X$ is locally compact, there is a compact neighbourhood $K$ at $x$ such that $K \cap \Omega^{(\epsilon, \tau)}(X)=\emptyset$. Take $y \in X$ and suppose that for every open neighbourhood $U$ at $y$ and for every $T \in \epsilon$, $(T \cdot U) \cap K \neq \emptyset$. Therefore there are a net $y_{\delta} \rightarrow y$ and an $\epsilon$-divergent net $t_{\delta}$ such that $t_{\delta} \cdot y_{\delta} \in K$. Since $K$ is compact, there are a subnet $y_{\delta_{i}} \rightarrow$ $y$ and a divergent subnet $t_{\delta_{i}}$ such that $t_{\delta_{i}} \cdot y_{\delta_{i}} \rightarrow u \in K$. This implies that $u \in K \cap \Omega^{(\epsilon, \tau)}(y) \subset K \cap \Omega^{(\epsilon, \tau)}(X)$, which contradicts the fact that $K \cap \Omega^{(\epsilon, \tau)}(X)=\emptyset$. Then, for every $y \in X$ there are $U \in \mathbf{t}_{X_{y}}$ and $T \in \epsilon$ such that $T \cdot U \cap K=\emptyset$. Since $X$ is regular, there is $V \in \mathbf{t}_{X_{x}}$ such that $\bar{V} \subset K$; then $X \backslash \bar{V}$ is $(\epsilon, \tau)$-exterior.

In this way, we obtain the following relation between bar-limits and omega-limits:

COROLlaRY 7.6. If $X$ is a locally compact regular flow, then

$$
\bar{L}^{(\epsilon, \tau)}(X)=\overline{\Omega^{(\epsilon, \tau)}(X)} .
$$

Proof. By Lemma 7.1, one has $\overline{\Omega^{(\epsilon, \tau)}(X)} \subset \bar{L}^{(\epsilon, \tau)}(X)$. Besides, if $x \notin$ $\overline{\Omega^{(\epsilon, \tau)}(X)}$, by Lemma 7.5 , there exists $V \in \mathbf{t}_{X_{x}}$ such that $X \backslash \bar{V}$ is $(\epsilon, \tau)$ exterior. By Proposition 7.3 it follows that $x \notin \bar{L}^{(\epsilon, \tau)}(X)$. 
We note that some of the main results (Theorem 6.13 and Corollary 6.14) proved in [19] are consequences of Corollary 7.6. Moreover, if we denote

$$
\begin{aligned}
& \operatorname{Poisson}^{\mathbf{l}}(X)=\operatorname{Poisson}^{-}(X), \\
& \operatorname{Poisson}^{\mathbf{r}}(X)=\operatorname{Poisson}^{+}(X), \\
& \operatorname{Poisson}^{\mathbf{c}}(X)=\operatorname{Poisson}^{+}(X) \cup \operatorname{Poisson}^{-}(X),
\end{aligned}
$$

we obtain the following results:

Corollary 7.7. If $X$ is a locally compact $T_{3}$ flow, then

(i) $L^{(\epsilon, \mathbf{d})}(X)=P(X) \subset$ Poisson $^{\epsilon}(X) \subset \Omega^{(\epsilon, \mathbf{d})}(X) \subset \overline{\Omega^{(\epsilon, \mathbf{d})}(X)}=$ $\bar{L}^{(\epsilon, \mathbf{d})}(X)$

(ii) $L^{(\epsilon, \mathbf{t})}(X)=A g^{(-\epsilon, \mathbf{d})}(X) \subset A g^{(-\epsilon, \mathbf{t})}(X) \subset \Omega^{(\epsilon, \mathbf{t})}(X) \subset \overline{\Omega^{(\epsilon, \mathbf{t})}(X)}=$ $\bar{L}^{(\epsilon, \mathbf{t})}(X)$.

Proof. (i) It follows from Theorem 6.3 and Corollary 7.6.

(ii) It is a consequence of Corollary 6.8, Corollary 6.10 and Corollary 7.6.

REMARK 7.8. If $X$ is a locally compact $T_{3}$ flow, then the following diagram is commutative, where all the arrows are inclusions:

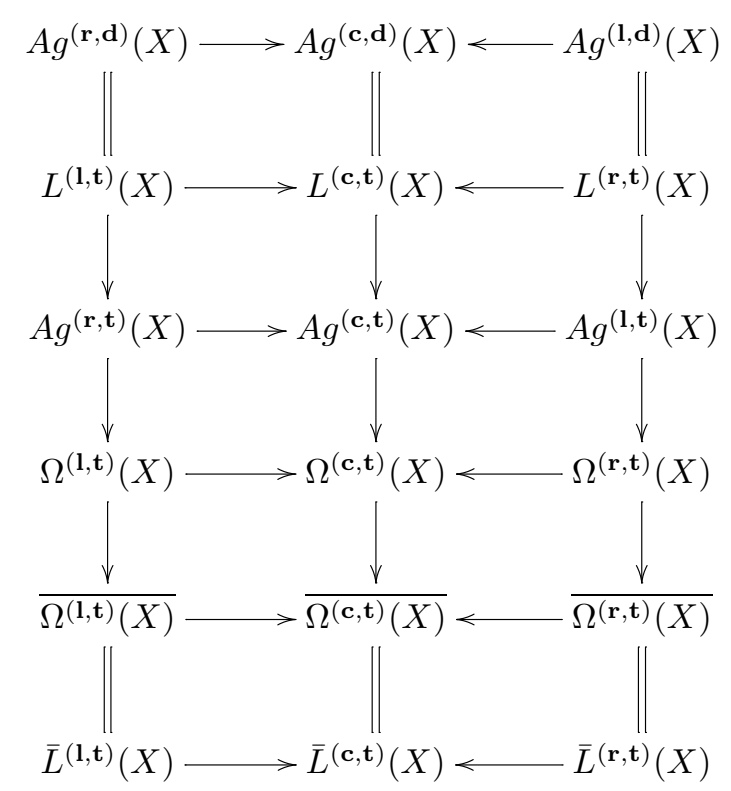

This diagram relates $(\epsilon, \mathbf{d})$-agglomerative points, $(-\epsilon, \mathbf{t})$-limits, $(\epsilon, \mathbf{t})$-agglomerative points, prolongational limits and $(-\epsilon, \mathbf{t})$-bar-limits. 
Remark 7.9. Given a flow $X$ and $x \in X$, we can say that $x$ is $(\epsilon, \tau)$ dispersive if $\Omega^{(\epsilon, \tau)}(x)=\emptyset$. We point out that $X \backslash A g^{(\epsilon, \tau)}(X)$ is the set of $(\epsilon, \tau)$ dispersive points of $X$. For $\tau=\mathbf{t}_{X}$ and $\epsilon=\mathbf{r}$, we can apply Theorems 1.8 and 2.6 in Chapter IV of [4] to prove that, under suitable conditions, $X \backslash A g^{(\epsilon, \tau)}(X)$ is a parallelizable subflow and $X \backslash L^{(-\epsilon, \tau)}(X)$ is an open parallelizable subflow.

Remark 7.10. We have seen in Remark 6.4 and Theorem 6.14 that the functors $L^{(\epsilon, \tau)}$ are idempotent under some topological conditions. In general the idempotent property for bar-limits $\bar{L}=\bar{L}^{(\epsilon, \tau)}$ could be more difficult to prove. However, we can always consider for the ordinals $1,2, \cdots, \alpha_{0}, \alpha_{0}+1, \cdots$ the corresponding injective maps:

$$
\cdots \rightarrow \bar{L}^{\alpha_{0}+1} \rightarrow \bar{L}^{\alpha_{0}} \rightarrow \cdots \rightarrow \bar{L}^{2} \rightarrow \bar{L}
$$

where $\bar{L}^{\beta+1}(X)=\bar{L}\left(\bar{L}^{\beta}(X)\right)$ and if $\beta$ is a limit ordinal $\bar{L}^{\beta}(X)=\bigcap_{\alpha<\beta} \bar{L}^{\alpha}(X)$. It is clear that for any flow $X$ there is an ordinal $\beta$ such that

$$
\bar{L}^{\beta+1}(X)=\bar{L}^{\beta}(X) .
$$

7.2. Limits, bar-limits and $(\epsilon, \tau)$-periodic points.

Definition 7.11. Let $(X, \varepsilon(X))$ be an $(\epsilon, \tau)$-exterior flow. A point $x \in X$ is said to be $(\epsilon, \tau)$-periodic if for every $U \in \mathbf{t}_{X_{x}}$, for every $T \in \epsilon$ and for every $V \in \tau_{x},(T \cdot V) \cap U \neq \emptyset$. Denote by $P^{(\epsilon, \tau)}(X)$ the $\tau$-closed subset of points of $X$ which are $(\epsilon, \tau)$-periodic.

Note that $x$ is an $(\epsilon, \tau)$-periodic point if and only if $x \in \Omega^{(\epsilon, \tau)}(x)$. Then, $P^{(\epsilon, \tau)}(X) \subset \Omega^{(\epsilon, \tau)}(X)$.

If $X$ is a flow, given $x \in X, x$ is an $(\epsilon, \tau)$-periodic point if $x$ is an $(\epsilon, \tau)$ periodic point in $X^{(\epsilon, \tau)} . P^{(\epsilon, \tau)}(X)$ denotes the subflow of $(\epsilon, \tau)$-periodic points of $X$.

EXAmPLe 7.12. Let $X=\mathbb{R}$ with the topology

$$
\mathbf{t}_{X}=\{(t,+\infty) \mid t \in \mathbb{R}\} \cup\{\emptyset, X\} .
$$

Consider the flow

$$
\varphi: \mathbb{R} \times X \rightarrow X, \quad \varphi(r, s)=r+s .
$$

Now it is easy to check that every $s \in X$ is an $(\mathbf{r}, \mathbf{d})$-periodic point and $X$ does not have $(\mathbf{l}, \mathbf{d})$-periodic points.

Lemma 7.13. Let $X$ be a flow and $x \in X$. Then, $x$ is $(\mathbf{l}, \mathbf{t})$-periodic if and only if $x$ is $(\mathbf{r}, \mathbf{t})$-periodic if and only $x$ is $(\mathbf{c}, \mathbf{t})$-periodic if and only if $x$ is a non-wandering point.

Proof. First, we prove that a non- $(\mathbf{l}, \mathbf{t})$-periodic point is a wandering point: Indeed, suppose that $x$ is a non- $(\mathbf{l}, \mathbf{t})$-periodic point; then there is $U \in \mathbf{t}_{X_{x}}$ and there is $T \in \mathbf{l}$ such that $(T \cdot U) \cap U=\emptyset$. Take $r_{0}^{\prime} \in \mathbb{R}$ such that $\left(-\infty, r_{0}^{\prime}\right] \subset T$; then, for every $r^{\prime} \leq r_{0}^{\prime}, \varphi_{r^{\prime}}(U) \cap U=\emptyset$. Since $\varphi_{r^{\prime}}$ is 
a homeomorphism, it follows that $U \cap \varphi_{-r^{\prime}}(U)=\emptyset$. Taking $r_{0}=-r_{0}^{\prime}$ one has that for $r \geq r_{0}, \varphi_{r}(U) \cap U=\emptyset$. Therefore $x$ is a wandering point. The converse and other implications can be proved similarly.

Consequently, one has that for any flow $X$ :

$$
N(X)=P^{(1, \mathbf{t})}(X)=P^{(\mathbf{c}, \mathbf{t})}(X)=P^{(\mathbf{r}, \mathbf{t})}(X) .
$$

Proposition 7.14. Suppose that $X$ is a flow. Then,

(i) $P(X) \subset P^{(\mathbf{l}, \mathbf{d})}(X) \cap P^{(\mathbf{r}, \mathbf{d})}(X)$,

(ii) $P^{(\mathbf{c}, \mathbf{d})}(X)=P^{(\mathbf{l}, \mathbf{d})}(X) \cup P^{(\mathbf{r}, \mathbf{d})}(X)$,

(iii) $P^{(\epsilon, \mathbf{d})}(X)=\operatorname{Poisson}^{\epsilon}(X)$,

(iv) $P^{(\epsilon, \mathbf{d})}(X) \subset P^{(\epsilon, \mathbf{t})}(X)$,

(v) $P^{(\epsilon, \mathbf{t})}(X)=N(X)$.

Proof. (i) is easy to check. To prove (ii), suppose that $x$ is $(\mathbf{c}, \mathbf{d})$ periodic and $x$ is not $(\mathbf{l}, \mathbf{d})$-periodic. Since $x$ is not $(\mathbf{l}, \mathbf{d})$-periodic, if $U$ is an open neighbourhood at $x$ and $T \in \mathbf{r}$, there are $T^{\prime} \in \mathbf{l}$ and $U^{\prime} \in \mathbf{t}_{X_{x}}$, satisfying that $U^{\prime} \subset U$ and $\left(T^{\prime} \cdot x\right) \cap U^{\prime}=\emptyset$. Taking into account that $T^{\prime \prime}=T^{\prime} \cup T \in \mathbf{c}$ and $x$ is $(\mathbf{c}, \mathbf{d})$-periodic, one has that $\left(T^{\prime \prime} \cdot x\right) \cap U^{\prime} \neq \emptyset$. This implies that $(T \cdot x) \cap U \neq \emptyset$; then $x$ is $(\mathbf{r}, \mathbf{d})$-periodic. Applying Lemma 7.13, we obtain (v) and the rest of the proof is straightforward.

REMARK 7.15. For a flow on a compact metric space, we have that the recurrent points in the sense of Birkoff (see Remark 3.6 of Chapter III in [4]) are $(\mathbf{c}, \mathbf{d})$-periodic points.

Proposition 7.16. Suppose that $X$ is a regular flow. If a point $x$ is not $(\epsilon, \mathbf{t})$-periodic, then there is $V \in \mathbf{t}_{X_{x}}$ such that $X \backslash \bar{V}$ is $(\epsilon, \mathbf{d})$-exterior. Moreover, there is $T \in \epsilon$ such that

$$
(T \cdot V) \subset X \backslash \bar{V} .
$$

Proof. If a point $x$ is not $(\epsilon, \mathbf{t})$-periodic, then there is an open neighbourhood $U$ at $x$ and there is $T \in \epsilon$ such that $(T \cdot U) \cap U=\emptyset$. Since $X$ is regular, there is $V \in \mathbf{t}_{X_{x}}$ such that $x \in V \subset \bar{V} \subset U$. Then $T \cdot V \subset X \backslash \bar{V}$. Let us check that $X \backslash \bar{V}$ is $(\epsilon, \mathbf{d})$-exterior:

Indeed, for a given $y \in X$, if $(\mathbb{R} \cdot y) \cap U \neq \emptyset$, then there is $r \in \mathbb{R}$ such that $r \cdot y \in U$. Since $(T \cdot U) \cap U=\emptyset$, it follows that $(r+T) \cdot(-r \cdot U) \cap U=\emptyset$. Taking $T^{y}=r+T$ and $U_{y}=-r \cdot U$, we have that $\left(T^{y} \cdot U_{y}\right) \cap U=\emptyset$. This implies that

$$
T^{y} \cdot y \subset T^{y} \cdot U_{y} \subset X \backslash \bar{V} .
$$

Otherwise, one has that $(\mathbb{R} \cdot y) \cap U=\emptyset$. Therefore $\mathbb{R} \cdot y \subset X \backslash \bar{V}$. Taking into account the definition of $(\epsilon, \mathbf{d})$-exterior, it follows that $X \backslash \bar{V}$ is $(\epsilon, \mathbf{d})$-exterior. 
THeOREM 7.17. If $X$ is a regular flow, then

$$
\bar{L}^{(\epsilon, \mathbf{d})}(X) \subset N(X) .
$$

Proof. By part (v) of Proposition 7.14, $P^{(\epsilon, \mathbf{t})}(X)=N(X)$. By Proposition 7.16, if $x \notin P^{(\epsilon, \mathbf{t})}(X)$, there is $V \in \mathbf{t}_{X_{x}}$ such that $X \backslash \bar{V}$ is $(\epsilon, \mathbf{d})$ - exterior. This implies that $x \notin \bar{L}^{(\epsilon, \mathbf{d})}(X)$.

Remark 7.18. If $X$ is a $T_{3}$ flow, then the following diagram is commutative, where all the arrows are inclusions:

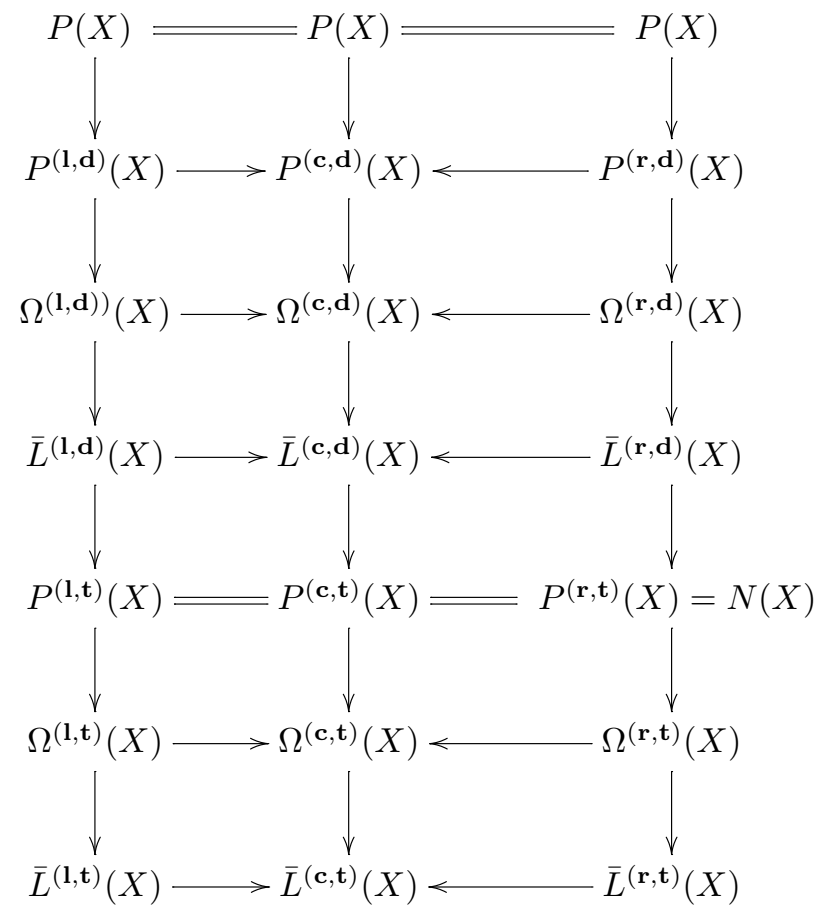

This diagram relates positively (negatively) Poisson-stable points, omegalimits, $(\epsilon, \mathbf{d})$-bar-limits, non-wandering points, first prolongational limits and $(\epsilon, \mathbf{t})$-bar-limits.

THEOREM 7.19. If $X$ is a $T_{3}$ locally compact flow and every nonwandering point is a Lagrange-stable point, then the following diagram is commutative, where all the arrows are inclusions (and we also have $\overline{\Omega^{(\epsilon, \tau)}(X)}=$ $\left.\bar{L}^{(\epsilon, \tau}(X)\right)$ : 


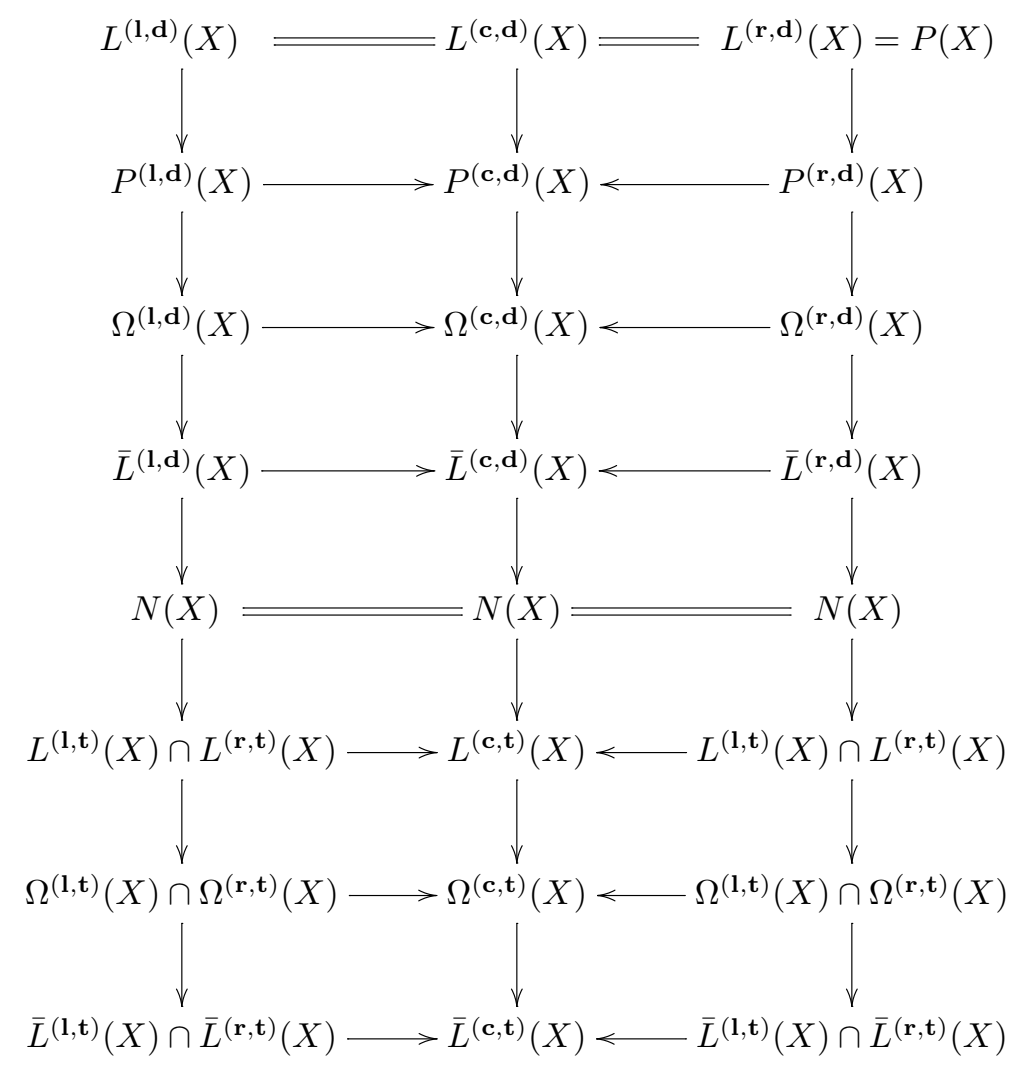

Proof. Notice that the results of Remark 7.18 can be completed by using Corollary 7.6 to obtain that $\overline{\Omega^{(\epsilon, \tau)}(X)}=\bar{L}^{(\epsilon, \tau)}(X)$. Moreover, from the condition that every non-wandering point is Lagrange-stable, applying Corollary 6.19 we also have that $N(X) \subset L^{(1, \mathbf{t})}(X) \cap L^{(\mathbf{r}, \mathbf{t})}(X)$.

Remark 7.20. We note that if $X$ is a $T_{2}$ compact flow, then $X$ is a $T_{3}$ locally compact and every point of $X$ is a Lagrange-stable point. In this case we have that $X=L^{(\epsilon, \mathbf{t})}(X)=\Omega^{(\epsilon, \mathbf{t})}(X)=\bar{L}^{(\epsilon, \mathbf{t})}(X)$ and all the subflows of the last three rows of diagram above are equal to $X$.

\section{Relations between limit spaces and Freudenthal end spaces}

Given a flow $X$, for $(\epsilon, \tau) \in\{\mathbf{l}, \mathbf{r}, \mathbf{c}\} \times\{\mathbf{d}, \mathbf{t}\}$ the corresponding $(\epsilon, \tau)$-limit and $(\epsilon, \tau)$-end spaces are related by the following commutative diagram: 


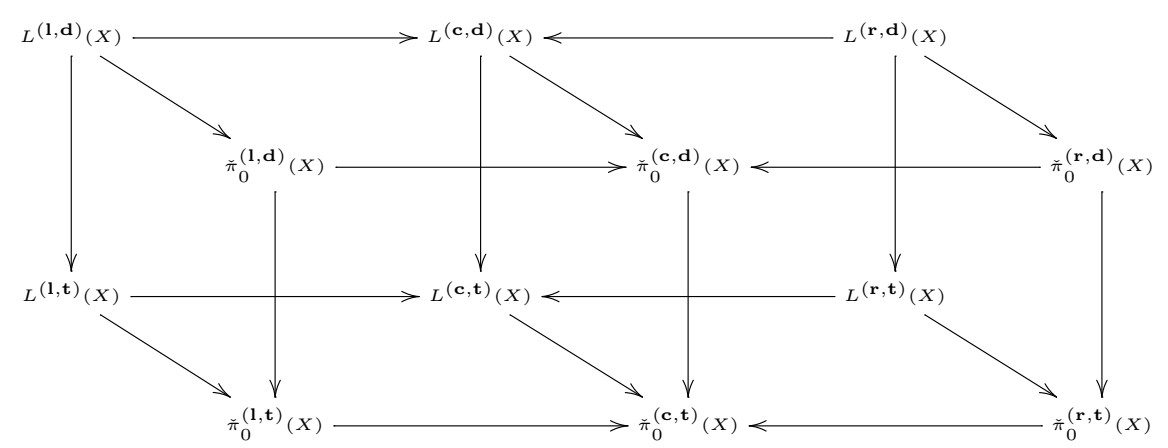

We have a similar diagram for $(\epsilon, \tau)$-bar-limit and $(\epsilon, \tau)$-bar-end spaces.

We note that this diagram gives decompositions of the different limits subflows as disjoint union of the fibres of the different canonical maps. Moreover, these various sets of Freudenthal ends can be used to divide the global flow as a union of disjoint basins associated with the end points of these Freudenthal sets.

It is interesting to observe the relationship between the limit of sets of path-components of exterior open subsets of an externology and the set of connected components of its limit. We can apply the properties of exterior spaces proved in Theorem 3.17 in [19] to the externologies $\varepsilon^{(\epsilon, \mathbf{d})}(X)$ (resp., $\left.\varepsilon^{(\epsilon, \mathbf{t})}(X)\right)$ of a flow $X$ to establish an isomorphism between the set of connected components of periodic points (resp., agglomerative points) and the profinite spaces of Freudenthal ends. Consequently, we have:

Corollary 8.1. Let $X$ be a non-empty flow and suppose that $X$ is a connected, locally path-connected space and $X^{(\epsilon, \tau)}$ is locally compact at infinity. Then,

(i) $L^{(\epsilon, \tau)}(X)=\bar{L}^{(\epsilon, \tau)}(X)$ is compact and $\check{\pi}_{0}^{(\epsilon, \tau)}(X)=\check{\bar{\pi}}_{0}^{(\epsilon, \tau)}(X)$,

(ii) the canonical map $L^{(\epsilon, \tau)}(X) \rightarrow \check{\pi}_{0}^{(\epsilon, \tau)}(X)$ is surjective (i.e., any Freudenthal end is representable),

(iii) $\check{\pi}_{0}^{(\epsilon, \tau)}(X)$ is a profinite compact space,

(iv) if $a \in \check{\pi}_{0}^{(\epsilon, \tau)}(X)$, then $L_{a}^{(\epsilon, \tau)}(X)$ is a non-empty continuum,

(v) if $\tau=\mathbf{t}_{X}$, then $\check{\pi}_{0}^{(\epsilon, \mathbf{t})}(X)=\{*\}$ has only one end and

$$
L_{*}^{(\epsilon, \mathbf{t})}(X)=L^{(\epsilon, \mathbf{t})}(X)=\bar{L}_{*}^{(\epsilon, \mathbf{t})}(X)
$$

is a non-empty continuum.

Given a topological space $Y$, we denote by $c(Y)$ the set of connected components of $Y$. Note that as a consequence of Corollary 8.1 we have:

Corollary 8.2. Let $X$ be a non-empty flow and suppose that $X$ is a connected, locally path-connected space and $X^{(\epsilon, \tau)}$ is locally compact at infinity. 
Then,

$$
c\left(L^{(\epsilon, \tau)}(X)\right) \cong \check{\pi}_{0}^{(\epsilon, \tau)}(X) .
$$

\section{CONClusions AND FURTher WORK}

In this paper we have seen that there are very nice connections between concepts of the theory of exterior spaces and the theory of continuous flows. These facts permit us to apply constructions and properties developed for exterior spaces to the study of dynamical systems.

On the one hand, for an exterior space, we have, among others, the following notions and constructions:

- notion of open and closed exterior subsets (Definition 2.1),

- the limit space (Definition 2.2),

- the bar-limit space (Definition 2.2),

- the set (or space) of Freudenthal end points (Subsection 2.1),

- the set (or space) of Freudenthal bar-end points (Subsection 2.1),

- the notion of locally compact at infinity (Definition 6.15),

- higher homotopy invariant of exterior spaces $([12,21])$ (Steenrod, Čech and Brown-Grossmann groups, see [10]).

On the other hand, for a continuous flow, we have common dynamical notions as the following:

- the omega-limit of a given point and the omega-limit of a subset of points (Definition 2.9),

- the first prolongational limit space of a given point and the first prolongational limit space of a subset of points (Definition 2.11 and Remark 2.13),

- periodic point (Subsection 2.2),

- positively (negatively) Poisson-stable point (Subsection 2.2),

- non wandering point (Definition 2.15),

- the region of attraction of a subset (Definition 2.18),

- attractor of a region (Definition 2.19),

- decomposition of a flow as disjoint union of basins (Definition 5.3),

- positively (negatively) agglomerative point (Definition 6.5),

- positively (negatively) Lagrange-stable point (Definition 6.18),

- dispersive and parallelizable flows (Remark 7.9), et cetera.

In our study we have found many interesting results relating the exterior notions and constructions given in the first list and the dynamic notions given in the second list. For example:

- Theorem 6.14 proves that the $(-\epsilon, \mathbf{t})$-limit of a $T_{2}$ flow is the region of attraction of the $(\epsilon, \mathbf{d})$-omega limit. 
- Theorem 7.19 summarizes many of the relations between the subflows associated to canonical exterior constructions and the more important subflows of a continuous dynamical system. In this Theorem we give some important relationships between $(\epsilon, \mathbf{d})$-limit, $(\epsilon, \mathbf{d})$-bar-limits, $(\epsilon, \mathbf{t})$-limits, $(\epsilon, \mathbf{t})$-bar limits and periodic points, Poison-stable points, omega-limits, non-wandering points, agglomerative points and first prolongational limits.

In addition, for a continuous flow $X$, the following important relationships and results have been proved in this paper:

- Given a point $x$ of a $T_{1}$ flow $X,\{x\}$ is an $(\epsilon, \mathbf{d})$-closed subset if and only if $x$ is a non-periodic point (Theorem 6.2);

- Given $x \in X,\{x\}$ is an $(\epsilon, \mathbf{t})$-closed subset if and only if $x$ is not a $(-\epsilon, \mathbf{d})$-agglomerative point (Theorem 6.9 and Definition 6.5);

- For $\epsilon \in\{\mathbf{l}, \mathbf{c}, \mathbf{r}\}$, the $(\epsilon, \mathbf{d})$-limit of a $T_{1}$ flow is the subset of periodic points (Theorem 6.3);

- For $\epsilon \in\{\mathbf{l}, \mathbf{c}, \mathbf{r}\}$, the $(\epsilon, \mathbf{t})$-limit of a $T_{1}$ flow is the subset of $(-\epsilon, \mathbf{d})$ agglomerative points of $X$ (Corollary 6.10);

- Results about the relation of the locally compact at infinity condition and attractors and repellers (Corollaries 6.16, 6.17 and Remark 6.20);

- Properties of $(\mathbf{l}, \mathbf{t})$-limit of a flow and the positively Lagrange-stable condition for a flow (Corollary 6.19);

- The $(\epsilon, \mathbf{d})$-bar-limit of a flow as the closure of the omega-limit of the flow (Corollary 7.6);

- The $(\epsilon, \mathbf{t})$-bar-limit of a flow as the closure of the first prolongational limit of the flow (Corollary 7.6);

- Remark 7.8 contains a diagram which relates $(\epsilon, \mathbf{d})$-agglomerative points, $(-\epsilon, \mathbf{t})$-limits, $(\epsilon, \mathbf{t})$-agglomerative points, prolongational limits and $(\epsilon, \mathbf{t})$-bar-limits;

- The complement of the $(\epsilon, \mathbf{t})$-limit of a flow and dispersive and parallelizable subflows (Remark 7.9);

- For a $T_{3}$ flow, the diagram given in Remark 7.18 relates positively (negatively) Poisson-stable points, omega-limits, $(\epsilon, \mathbf{d})$-bar-limits, nonwandering points, first prolongational limits and $(\epsilon, \mathbf{t})$-bar-limits;

- Freudenthal ends of an exterior space and decomposition of a flow as disjoint union of basins (Proposition 5.2 and Definition 5.3);

- The condition of locally compact at infinity is related to the decomposition of set of periodic points or the set of agglomerative points as disjoint union of continuum components (Corollary 8.1);

- The isomorphism obtained (under the conditions of Corollary 8.2) between the space of connected components of the subflow of periodic 
points or the set of connected components of the subflow of agglomerative points and the corresponding set of Freudenthal ends.

With respect to further work and new research lines relating exterior spaces and continuous flows we suggest the following:

- To use higher homotopy invariant of exterior spaces (Steenrod, Cech and Brown-Grossmann groups) as new tools in the analysis of local and global stability properties of continuous flows and in the study of chaotic dynamical systems.

- To study the role of the externologies $\varepsilon^{\left(\epsilon, \tau_{\text {int }}\right)}(X)$, where $\tau_{\text {int }}$ is the intrinsic topology, in the study of repellers and atractors of a dynamical system. In particular, our techniques based on exterior flows can be used to generalize some results obtained in $[1-3]$.

- To study the role of the externologies $\varepsilon^{\left(\epsilon, \tau_{\text {tr }}\right)}(X)$ with respect to the dynamical properties of a flow (without fixed points) in a Riemannian manifold, where $\tau_{\text {tr }}$ is the topology of the foliation transverse to the flow.

- To discover the existence of new relationships between the theory of exterior spaces and the theories of discrete and continuous dynamical systems.

\section{ACKNOWLEDGEMENTS.}

Partially supported by Ministerio de Educación y Ciencia (grant MTM2013 -41768-P) and University of La Rioja (projects: APPI16/03, EGI16/42).

\section{REFERENCES}

[1] H. Barge and J.M.R. Sanjurjo, Unstable manifold, Conley index and fixed points of flows, J. Math. Anal. Appl. 420 (2014), 835-851.

[2] H. Barge and J.M.R. Sanjurjo, Regular blocks and Conley index of isolated invariant continua in surfaces, Nonlinear Anal. 146 (2016), 100-119.

[3] H. Barge and J.M.R. Sanjurjo, Fixed points, bounded orbits and attractors of planar flows, A mathematical tribute to Professor José María Montesinos Amilibia, Dep. Geom. Topol. Fac. Cien. Mat. UCM, Madrid, 2016, 125-132.

[4] N.P. Bhatia and G. P. Szegö, Stability theory of dynamical systems, Springer-Verlag, Berlin-Heidelberg-New York, 1970.

[5] G.D. Birkhoff, Dynamical systems, AMS, Colloquium Publications, vol. 9, 1927.

[6] K. Borsuk, Concerning homotopy properties of compacta, Fund. Math. 6 (1968), 223254.

[7] K. Borsuk, Concerning the notion of the shape of compacta, 1969 Proc. Internat. Sympos. on Topology and its Applications (Herceg-Novi, 1968), Savez Društava Mat. Fiz. i Astronom., Beograd 1969, 98-104.

[8] M. Cárdenas, F. F. Lasheras and A. Quintero, Detecting cohomology classes for the proper LS category. The case of semi-stable 3-manifolds, Math. Proc. Cambridge Philos. Soc. 152 (2012), 223-249.

[9] A. Del Río, L.J. Hernández and M.T. Rivas, S-types of global towers of spaces and exterior spaces, Appl. Categ. Structures 17 (2009), 287-301. 
[10] D. Edwards and H. Hastings, Čech and Steenrod homotopy theories with applications to geometric topology, Lect. Notes Math. 542, Springer, 1976.

[11] J.I. Extremiana, L.J. Hernández and M.T. Rivas, Sobre el infinito de los espacios, Proceedings of the workshop on proper homotopy theory, Univ. La Rioja, 1993, 23-69.

[12] J.I. Extremiana Aldana, L.J. Hernández Paricio and M.T. Rivas Rodriguez, Postnikov factorizations at infinity, Topology Appl. 153 (2005), 370-393.

[13] H. Freudenthal, Über die Enden topologisher Räume und Gruppen, Math. Z. 33 (1931), 692-713.

[14] J.M. García-Calcines, P.R. García-Díaz and A. Murillo Mas, A Whitehead-Ganea approach for proper Lusternik-Schnirelmann category, Math. Proc. Cambridge Philos. Soc. 142 (2007), 439-457.

[15] J.M. García-Calcines, P.R. García-Díaz and A. Murillo, The Ganea conjecture in proper homotopy via exterior homotopy theory, Math. Proc. Cambridge Philos. Soc. 149 (2010), 75-91.

[16] J.M. García-Calcines, M. García-Pinillos and L.J. Hernández, A closed simplicial model category for proper homotopy and shape theories, Bull. Austral. Math. Soc. 57 (1998), 221-242.

[17] J.M. García-Calcines, M. García-Pinillos and L.J. Hernández, Closed simplicial model structures for exterior and proper homotopy, Appl. Categ. Structures 12 (2004), 225243.

[18] J.M. García-Calcines and L.J. Hernández, Sequential homology, Top. and its Appl. 114 (2001), 201-225.

[19] J.M. García Calcines, L.J. Hernández Paricio and M.T. Rivas Rodriguez, Limit and end functors of dynamical systems via exterior spaces, Bull. Belg. Math. Soc. Simon Stevin 20 (2013), 937-959.

[20] J.M. García Calcines, L.J. Hernández Paricio and M.T. Rivas Rodriguez, A completion construction for continuous dynamical systems, Topol. Methods Nonlinear Anal. 44 (2014), 497-526.

[21] M. García-Pinillos, L.J. Hernández Paricio and M.T. Rivas Rodriguez, Exact sequences and closed model categories, Appl. Categ. Structures 18 (2010), 343-375.

[22] L.J. Hernández Paricio, Applications of simplicial $M$-sets to proper and strong shape theories, Trans. Amer. Math. Soc. 347 (1995), 363-409.

[23] B. Kerékjártó, Vorlesungen über Topologie, Springer-Verlag, vol.1, 1923

[24] E.N. Lorenz, Deterministic nonperiodic flow, J. Atmos. Sci. 20, (1963), 130-141.

[25] J. Milnor, Morse theory, Princeton University Press, Princeton, 1963.

[26] T. Porter, Proper homotopy theory, Handbook of Algebraic Topology, North-Holland, Amsterdam, 1995, 127-167.

[27] J.W. Robbin and D. Salamon, Dynamical systems, shape theory and the Conley index, Ergodic Theory Dynam. Systems 8 (1988), Charles Conley Memorial Volume, 375-393.

[28] J.J. Sánchez-Gabites, Dynamical systems and shapes, Rev. R. Acad. Cienc. Exactas Fs. Nat. Ser. A Math. RACSAM 102 (2008), 127-159.

[29] J.M. Sanjurjo, Morse equations and unstable manifolds of isolated invariant sets, Nonlinearity 16 (2003), 1435-1448.

[30] L.C. Siebenmann, The obstruction to finding a boundary for an open manifold of dimension greater than five, Thesis, 1965. http://www.math.uchicago.edu/ shmuel/ tom-readings/.

[31] S. Smale, Differentiable dynamical systems, Bull. Amer. Math. Soc. 73 (1967), 747817. 
J. M. G. Calcines

Departamento de Matemáticas, Estadística e I.O.

Universidad de La Laguna

38200 La Laguna

Spain

E-mail: jmgarcal@ull.es

L. J. Hernández Paricio

Departamento de Matemáticas y Computación

Universidad de La Rioja

26006 Logroño

Spain

E-mail: luis-javier.hernandez@unirioja.es

M. T. Rivas Rodríguez

Departamento de Matemáticas y Computación

Universidad de La Rioja

26006 Logroño

Spain

E-mail: maria-teresa.rivas@unirioja.es

Received: 14.10 .2016 .

Revised: 12.1.2017. 